\title{
Magnetic Phase Diagram of Nanostructured Zinc Ferrite as a Function of Inversion Degree $\delta$
}

M A Cobos ${ }^{a, b}$, P de la Presa ${ }^{a, c, *}$, I. Llorente ${ }^{b}$, JM Alonso $^{a, d}$, A García-Escorial $^{b}, P$ Marín $^{a, c}$, A. Hernando ${ }^{a, c, e}, J$ A Jiménez $z^{b}$.

a. Instituto de Magnetismo Aplicado (UCM-ADIF-CSIC), A6 22,500 Km, 28260 Las Rozas, Spain

b. Centro Nacional de Investigaciones Metalúrgicas (CENIM-CSIC), Avda. Gregorio del Amo, 8, 28040 Madrid, Spain

c. Dpto Física de Materiales, UCM, Ciudad Universitaria, 28040 Madrid, Spain

d. Instituto de Ciencias de Materiales de Madrid (ICMM-CSIC), Avda Sor Juana Inés de la Cruz 8, 28040 Madrid, Spain

e. Donostia International Physics Center DIPC, Paseo Manuel de Lardizabal 4, 2018, Donostia-San Sebastián, Spain

* Corresponding author: Patricia de la Presa: pmpresa@ucm.es 
ABSTRACT. Magnetic properties of spinel zinc ferrite are strongly linked to the synthesis method and the processing route since they control the microstructure of the resulting material. In this work, $\mathrm{ZnFe}_{2} \mathrm{O}_{4}$ nanoparticles were synthetized by mechanochemical reaction of stoichiometric $\mathrm{ZnO}$ and $\alpha-\mathrm{Fe}_{2} \mathrm{O}_{3}$, and single phase $\mathrm{ZnFe}_{2} \mathrm{O}_{4}$ was obtained after $150 \mathrm{~h}$ milling. The as-milled samples, with a high inversion degree, were subjected to different thermal annealing up to $600{ }^{\circ} \mathrm{C}$ in order to control the inversion degree and, consequently, the magnetic properties. The as-milled samples, with a crystallite size of $11 \mathrm{~nm}$ and inversion degree $\delta=0.57$, showed ferrimagnetic behavior even above room temperature, as shown by Rietveld refinements of XRD pattern and SQUID magnetometry. The successive thermal treatments at 300, 400, 500 and $600{ }^{\circ} \mathrm{C}$ made $\delta$ decreases from 0.57 to 0.18 affecting the magnetic properties. A magnetic phase diagram as a function of $\delta$ can be inferred from the results: for $\delta<0.25$, antiferromagnetism, ferrimagnetism and spin frustration were observed to coexist; for $0.25<\delta<0.5$, the ferrimagnetic clusters coalesced and spin glass behavior vanished, remaining only a pure ferrimagnetic phase with a maximum magnetization of $M_{s}=3.5 \mu_{B}$. Finally, for $\delta>0.5$, a new antiferromagnetic order appeared due to the overpopulation of non-magnetic $\mathrm{Zn}$ on octahedral sites that leads to an equally distributed magnetic cations in octahedral and tetrahedral sites. 


\section{Introduction}

Ferrites are a group of ceramic materials extensively used for the industry due to their electrical and magnetic properties, ${ }^{1-3}$ which can be easily tuned depending on the choice, amount and oxidation state of metal ions, specific features of cation arrangement in the crystal lattice and the processing route used for the fabrication. ${ }^{4-8}$ Although the generic term ferrites includes several groups of magnetic compounds consisting of iron oxide combined with one or more other metals, the most common group is the spinel ferrite. These spinel have the formula $\mathrm{MFe}_{2} \mathrm{O}_{4}$, where $\mathrm{M}$ is usually one or more divalent or trivalent metals, but it can also represent other cations with different charges, as long as positive charge are compensated for neutrality of the unit cell. ${ }^{9}$ An ideal spinel structure is composed by 32 oxygen anions packed in a face centered cubic (FCC) structure. This oxygen FCC arrangement leaves 64 tetrahedral coordinated sites and 32 octahedral coordinated sites in the unit cell, but only one eighth of the tetrahedral (A) and one half of the octahedral (B) sites are occupied with cations. As the distribution of metal ions on the $\mathrm{A}$ and $\mathrm{B}$ sites is determined by a large number of energy terms (Coulomb energy, Bohr repulsion energy, ordering energy, polarization energy of the anion, individual preference of ions for occupying tetrahedral or octahedral sites as result of their electronic configuration, etc), it is usually very difficult (or in fact impossible) to predict which distribution could materialize. In normal spinel ferrites, the A sites are filled by the divalent ions like $\mathrm{Mg}^{2+}, \mathrm{Mn}^{2+}, \mathrm{Cd}^{2+}$ and $\mathrm{Zn}^{2+}$, leaving all the $\mathrm{Fe}^{3+}$ ions on the $\mathrm{B}$ sites. ${ }^{10}$ On the other hand, in inverse spinel ferrites, divalent metals like $\mathrm{Co}^{2+}, \mathrm{Fe}^{2+}, \mathrm{Cu}^{2+}$ and $\mathrm{Ni}^{2+}$ are commonly occupying the $\mathrm{B}$ sites and $\mathrm{Fe}^{3+}$ ions are equally divided between $\mathrm{A}$ and $\mathrm{B}$ sites. These cation distributions represent extreme cases. In general, the ionic distribution is mixed and it can be represented by $\left[\mathrm{M}_{1}\right.$ $\left.{ }_{\delta} \mathrm{Fe}_{\delta}\right]^{\mathrm{A}}\left[\mathrm{M}_{\delta} \mathrm{Fe}_{2-\delta}\right]^{\mathrm{B}} \mathrm{O}_{4}$, where $\delta$ is the inversion parameter, which specifies the fraction of $\mathrm{Fe}^{+3}$ ions in A-sites. ${ }^{11}$ Accordingly, $\delta=0$ and 1 stand for the normal and inverse cases, respectively.

Among spinel, zinc ferrites have been one of the most widely studied systems. Traditionally, zinc ferrites have been mainly used as a synthetic inorganic pigment for coloring plastics and rubber and for preparing high corrosion resistant organic coating systems, ${ }^{12}$ and, in recent years, the attention is paid on different applications like gas sensor, ${ }^{13}$ photo catalytic disinfection, ${ }^{14}$ or photo catalytic degradation of different chemical species. ${ }^{14-17}$ In addition, an interesting property of this material is the 
possibility to tune their magnetic behavior by varying the particle and/or crystallite size. In this sense, the change from paramagnetic to superparamagnetic or ferrimagnetic behavior at room temperature for nanosized zinc ferrite has been associated to the partial exchange at the spinel lattice between $\mathrm{Fe}^{3+}$ ions in the octahedral sites and $\mathrm{Zn}^{2+}$ ions in the tetrahedral sites. ${ }^{18-20}$ This mixed cation distribution is favored in nanosized zinc ferrite, but comparing magnetic properties of nanoparticles prepared by different synthesis methods, as co-precipitation, ${ }^{21}$ sol-gel, ${ }^{22}$ hydrothermal route, ${ }^{23}$ combustion, ${ }^{24}$ forced hydrolysis in a polyol medium, ${ }^{25}$ mechanochemical synthesis, ${ }^{26-29}$ among others, it can be concluded that they depend not only on the particle size but also on the synthesis method. Whereas $\mathrm{ZnFe}_{2} \mathrm{O}_{4}$ in bulk shows antiferromagnetic (AFM) to paramagnetic $(\mathrm{PM})$ transition at $\mathrm{T}_{\mathrm{N}} \approx 9 \mathrm{~K}$, particles prepared by nonequilibrium processing present a transition to ferromagnetic (FiM) or ferromagnetic (FM) ordering at significantly higher temperatures: around $30 \mathrm{~K}$ for the coprecipitation ${ }^{30}$ and the solgel methods ${ }^{31}$, above $77 \mathrm{~K}$ for the ball-milled nanoparticles, ${ }^{32}$, or near room temperature in thin film prepared by a sputtering method. ${ }^{33}$

It has been reported elsewhere that materials synthetized by high-energy milling present a significant amount of structural and microstructural defects ${ }^{34-35}$ and thus, in combination with thermal treatments, a huge assortment of microstructures exhibiting new and unusual properties can be produced. The advantage of this technology is that the powder can be produced in large quantities and the processing and annealing parameters can be carefully controlled to produce the desired inversion degree and to reduce strains and structural defects introduced by milling. For this goal, it is crucial to determine changes on microstructural parameters like the lattice constant, inversion degree, crystallite size and lattice strains (or microstrain) as well as to establish their correlation with the magnetic properties.

This work is aimed to understand the relation between microstructure and magnetic properties of nanosized zinc ferrites and to explore the capability of tailoring their magnetic properties through the processing route. Zinc ferrite has been synthesized by high-energy ball milling starting from $\mathrm{ZnO}$ and $\alpha-\mathrm{Fe}_{2} \mathrm{O}_{3}$ and the phase evolution is investigated as a function of milling time. Pure $\mathrm{ZnFe}_{2} \mathrm{O}_{4}$ with high inversion degree $(\delta \approx 0.6)$ is obtained after $150 \mathrm{~h}$ milling, and afterwards, the inversion degree is modified by thermal treatments at different temperatures to finally obtain a very low inversion 
degree $(\delta \approx 0.15)$. The analysis of structure and the magnetic properties of the annealed samples allow to determine a magnetic phase diagram as a function of the inversion degree $\delta$.

\section{Experimental procedure}

Stoichiometric $\mathrm{ZnFe}_{2} \mathrm{O}_{4}$ nanoparticles were synthetized by mechanochemical reaction in air a powder mixture of $\mathrm{ZnO}$ and $\alpha-\mathrm{Fe}_{2} \mathrm{O}_{3}$ (both $99 \%$ purity) supplied by Alpha Aesar in a 1:1 molar ratio. A total amount of $10 \mathrm{~g}$ of the oxides together with stainless steel balls of $10 \mathrm{~mm}$ were introduced into a stainless steel jar of $125 \mathrm{~cm}^{3}$ in ball-to-powder weight ratio of 10:1. The milling process was carried out up at room temperature in a planetary ball mill Retsch PM4 working at an average rotation speed of $275 \mathrm{rpm}$. The milling process was interrupted at selected time from 5 up to $150 \mathrm{~h}$ and a small amount of powder was removed to determine the optimal milling time from X-ray diffraction (XRD) measurements and to follow the evolution of their particle size and morphology with a Hitachi S-4800 FEG-SEM microscope. Finally, the powder milled for $150 \mathrm{~h}$, where a single phase was identified, was annealed for $1 \mathrm{~h}$ at temperatures ranging from 300 to $600{ }^{\circ} \mathrm{C}$ to eliminate strains and structural defects introduced by milling in addition to modify the inversion degree which have a strong contribution on the magnetic properties.

Microstructural evolution as a function of the milling time and annealing treatments was characterized by X-ray diffraction (XRD). XRD measurements were conducted with a Co radiation in a Bruker AXS D8 diffractometer equipped with a Goebel mirror and a LynxEye detector. XRD spectra were collected over a range from 10 to $120^{\circ}$ in BraggBrentano geometry with a step width of $0.01^{\circ}$. We have used the version 4.2 of Rietveld analysis program TOPAS (Bruker AXS) to model the full pattern with the crystallographic information of $\mathrm{ZnO}, \alpha-\mathrm{Fe}_{2} \mathrm{O}_{3}$ and $\mathrm{ZnFe}_{2} \mathrm{O}_{4}$ obtained from Pearson's Crystal Structure databases. ${ }^{36}$ The quantitative results showed typical standard deviation of about $1 \%$. Beside global parameters like background, zero displacement, scale factors and peak breadth, the refinement protocol also included structure parameters for the zinc ferrite. Isotropic temperature factors $\left(\mathrm{B}_{\text {iso }}\right)$ used in Rietveld refinements were fixed to the values reported by O'Neill. ${ }^{37}$ As the unit-cell parameters $a$ and oxygen fractional coordinates $(x, y, z)$ define the resulting tetrahedral $(\mathrm{T}-\mathrm{O})$ and octahedral $(\mathrm{M}-\mathrm{O})$ bond lengths, and the cation distribution determines the site-scattering in terms of equivalent 
electrons (i.e., mean atomic number), structure parameters optimized during the Rietveld refinement where $a, x=y=z$ and the degree of inversion $\delta$ (constraining the octahedral and tetrahedral sites to keep the sum of the same cations in the two sites to its stoichiometric value). Uncertainties in these three parameters were determined from the standard deviation obtained from the least-squares refinements.

Although the determination of crystallite size and lattice strain simultaneously from line broadening is not trivial, Balzar et $\mathrm{al}^{38}$ have shown that modeling line profiles with multiple Voigt functions can work reasonably well for line-broadening analysis. Thus, the double Voigt approach was used in this work for the size-strain separation. For this analysis, the instrumental contribution to peak broadening was removed with the breadth and shape parameters derived from the diffraction patter of a corundum sample. However, the limited reliability of these results can be inferred form the large standard deviation for domain size and strain parameters (ranging from 10 to 20\%).

Contamination from the milling medium in powders processed by ball milling has been reported elsewhere. ${ }^{34}$ To investigate the contamination with $\mathrm{Fe}, \mathrm{Ni}$ and $\mathrm{Cr}$ by the friction of the particles with milling balls and jar walls, the chemical composition of milled powders was determined with a FISCHERSCOPE X-RAY XUV energy dispersive X-ray fluorescence spectrometer (EDXRF). The transmitted electron microcopy technique has been used to determine the particle size of the as-milled particles. The images used for this task were obtained using a JEOL 3000F. In order to avoid measurements error during image analysis, the recognition and delimitation of primary particles in agglomerates was performed manually considering that they are both small and have a smooth surface.

Magnetic characterization of the samples as a function of applied field and temperature has been carried out using a standard superconducting quantum interference device (SQUID) MPMS (from Quantum Design) magnetometer with the maximum applied field of 5 T. In addition, Physical Property Measurement System (PPMS®) Quantum Design Vibrant sample magnetometer (VSM) was also used. Hysteresis cycles up to $5 \mathrm{~T}$ have been measured at 5, 100 and $300 \mathrm{~K}$. Zero field cooled (ZFC) and field cooled (FC) measurements were made at applied field of 50 Oe between 5 and $300 \mathrm{~K}$. 
For the investigation of spin-glass like behavior, aging and memory experiments were performed under FC protocols in the samples annealed at 600 and $500{ }^{\circ} \mathrm{C}$. The memory effect is an experimental signature of spin glass systems. ${ }^{39-42}$ In the FC memory protocol, the sample was cooled in a magnetic field of 50 Oe with intermittent stops at $100,75,50,35$ and $20 \mathrm{~K}$. At each stop, the field was switched off for $10^{4} \mathrm{~s}$, and then returned to 50 Oe before resuming cooling.

\section{Results}

\subsection{Microstructural characterization}

$\mathrm{XRD}$ is a particularly useful tool for monitoring the chemical and structural transformations induced in the precursor's mixture by milling since it is a rapid and non-destructive technique, and it requires minimal sample preparation. XRD patterns of the samples obtained at different milling time are shown in Fig. 1A to illustrate the mechanically induced evolution of the $\mathrm{Fe}_{2} \mathrm{O}_{3} / \mathrm{ZnO}$ mixture submitted to the high-energy ball-milling process. After $5 \mathrm{~h}$ milling, it is observed only a decrease of the peak height and a noticeable broadening of all reflections corresponding to ZnO (JCPDS 36-1451) and hematite (JCPDS 33-0664), that is associated to a decrease of the crystallite size and an accumulation of microstrain during milling. After $20 \mathrm{~h}$ of milling, broad peaks of a new phase are observed (JCPDS 22-1012) corresponding to the $\mathrm{ZnFe}_{2} \mathrm{O}_{4}$. Further milling leads to a progressive formation of zinc ferrite manifested by the gradual increase in the intensity of the diffraction peaks of this phase and the progressive decrease of the reflections corresponding to $\alpha-\mathrm{Fe}_{2} \mathrm{O}_{3}$ and $\mathrm{ZnO}$. After $110 \mathrm{~h}$ of milling, the main phase is zinc ferrite and those corresponding to $\mathrm{ZnO}$ have completely disappeared, whereas some reflections of hematite are still present; additionally, $\sim 1 \%$ of $\alpha-F e$ coming probably from the jar appears. Finally, after $150 \mathrm{~h}$ of milling only the reflections of nanocrystalline $\mathrm{ZnFe}_{2} \mathrm{O}_{4}$ are observed and the material can be considered as pure zinc ferrite plus the jar contamination of $1 \% \alpha-\mathrm{Fe}$.

The amount of the different phases presents during the ball milling and the most important microstructural parameter of zinc ferrite are determined using Rietveld refinement. The obtained results are summarized in Fig. 1B, Table 1 and Fig S1 (supporting information). This table shows that the crystallite size for zinc ferrite remains essentially constant for all samples, and only lattice parameter changes with 
increasing milling time up to $150 \mathrm{~h}$. On the other hand, the inversion degree of the zinc ferrite defined as the fraction of A-sites occupied by $\mathrm{Fe}^{3+}$, reaches a value close to 0.6 in all samples (see Table 1), suggesting the mechanosynthesized zinc ferrites present a random distribution of both $\mathrm{Fe}^{3+}$ cations between $\mathrm{A}$ and $\mathrm{B}$ sites as soon as the ferrite phase is formed.
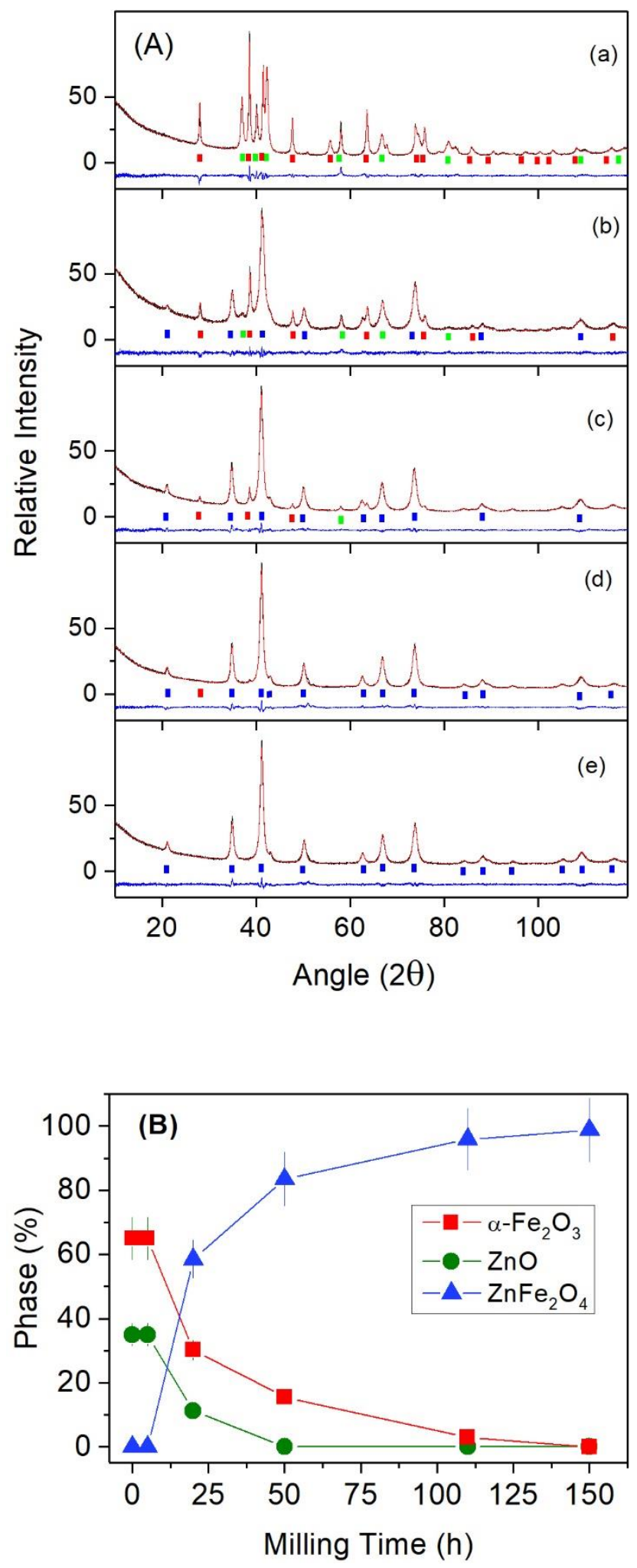
Figure 1: (A) XRD patterns of the $\mathrm{Fe}_{2} \mathrm{O}_{3} / \mathrm{ZnO}$ mixture after high-energy ball-milling for (a) $5 \mathrm{~h}$, (b) $20 \mathrm{~h}$, (c) $50 \mathrm{~h}$ (d) $110 \mathrm{~h}$ and (e) $150 \mathrm{~h}$. Red, green and blue bars peaks, correspond to hematite, zincite and ferrite phase peaks, respectivily. The red line is the experimental data, black line is the fit, and blue line is the difference. (B) Phase evolution with milling time.

Table 1. Composition, structural and microstructural parameters obtained after Rietveld refinement of the XRD patterns. The milling time in hours, the amount of the ferrite phase as a function of milling time, the crystallite size with milling time, the inversion degree, the lattice parameters, the $\mu$-deformation and the coordinates $(\mathrm{x}, \mathrm{y}, \mathrm{z})$ of oxygen atoms, are given in the table. The numbers in parenthesis represent the standard deviations.

\begin{tabular}{|c|c|c|c|c|c|c|}
\hline $\begin{array}{c}\text { Milling } \\
\text { time } \\
(\mathbf{h})\end{array}$ & $\begin{array}{c}\text { Ferrite } \\
\text { Phase } \\
(\boldsymbol{\%})\end{array}$ & $\begin{array}{c}\text { Crystal. } \\
\text { size } \\
(\mathbf{n m})\end{array}$ & $\begin{array}{c}\text { Inversion } \\
\text { parameter } \\
(\boldsymbol{\delta})\end{array}$ & $\begin{array}{c}\text { Lattice } \\
\text { parameter } \\
(\mathbf{A})\end{array}$ & $\begin{array}{c}\boldsymbol{\mu} \text {-deformation } \\
(\boldsymbol{\varepsilon})\end{array}$ & $\begin{array}{c}\text { O-Position } \\
(\mathbf{x}=\mathbf{y}=\mathbf{z})\end{array}$ \\
\hline 10 & $5.4(5)$ & $8.4(8)$ & $0.57(1)$ & $8.4409(5)$ & $0.00010(2)$ & $0.254(2)$ \\
\hline 20 & $58.5(6)$ & $8.5(8)$ & $0.60(1)$ & $8.4426(5)$ & $0.0007(1)$ & $0.249(2)$ \\
\hline 50 & $84.5(8)$ & $10(1)$ & $0.57(1)$ & $8.4435(5)$ & $0.0016(2)$ & $0.244(2)$ \\
\hline 70 & $84.4(8)$ & $10(1)$ & $0.56(1)$ & $8.4421(5)$ & $0.0020(2)$ & $0.244(2)$ \\
\hline 110 & $96.8(9)$ & $11(1)$ & $0.58(1)$ & $8.4363(5)$ & $0.0015(2)$ & $0.244(2)$ \\
\hline 150 & 100 & $11(1)$ & $0.57(1)$ & $8.4323(5)$ & $0.0015(2)$ & $0.245(2)$ \\
\hline
\end{tabular}

After $150 \mathrm{~h}$, milled powders consisted of cabbage-like particulate clusters as shown in Fig 2A. At higher magnifications, these agglomerates exhibit small and smooth spherical features with high crystallinity degree that corresponds to of primary $\mathrm{ZnFe}_{2} \mathrm{O}_{4}$ nano-particles, as observed in Fig 2B. In order to avoid measurements error during image analysis, the recognition and delimitation of primary particles in agglomerates was performed manually. The size distribution of these particles was obtained by the histograms presented in inset of Fig 2A, in which sizes are plotted on the x-axis, incremental sizes are represented by the width of bars in the histogram, while the calculated relative frequencies are shown in the vertical column (y-axis). Statistical quantitative analysis performed directly from the data represented in this graphic yielded an average particle size of $11.5 \mathrm{~nm}$ and a standard deviation of $2.0 \mathrm{~nm}$. This low value of the polydispersity suggests that the mechanical alloying can produce rather homogenous ferrite nanoparticles highly aggregated. More TEM images are presented in Fig. S2 from supporting information. 


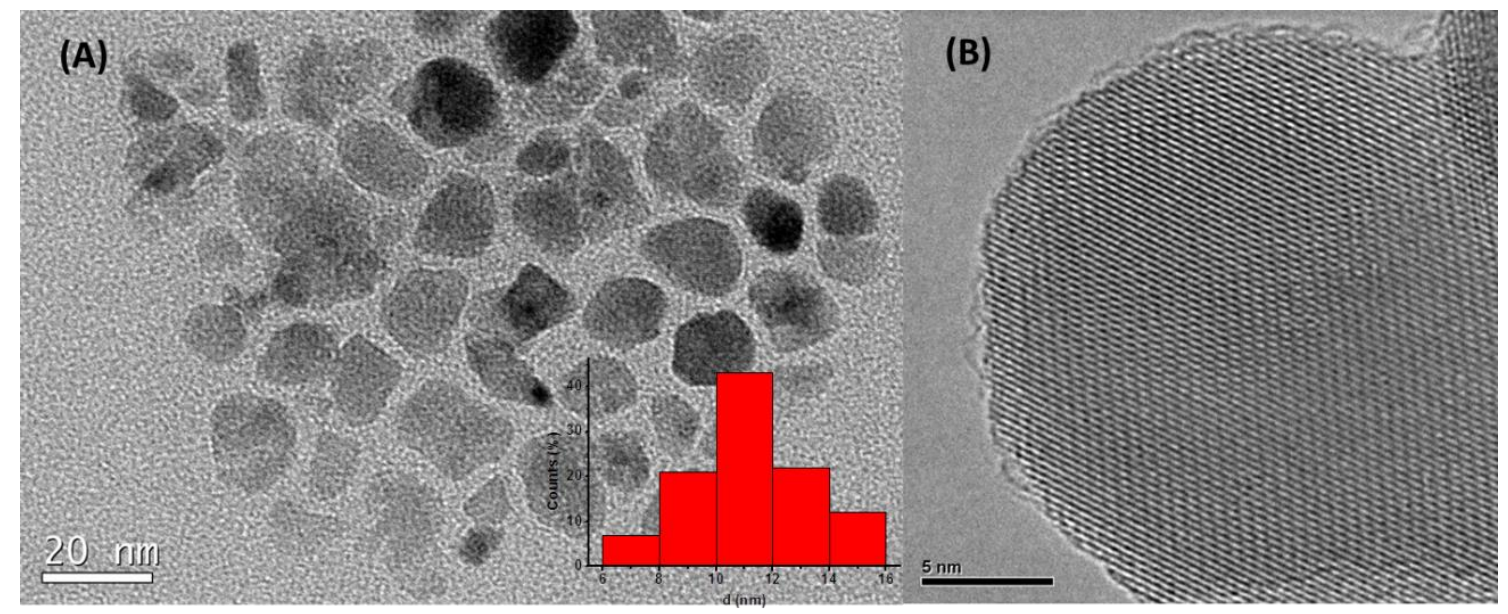

Figure 2: TEM micrographs of (A) powders milled for $150 \mathrm{~h}$ and (B) the HRTEM image of a single particle (A). The inset in (A) shows the particle size distribution with an average diameter $11.5 \mathrm{~nm}$ and discrete standard deviation $\sigma=2.0$.

Contamination by $\mathrm{Fe}, \mathrm{Cr}$ and $\mathrm{Ni}$ is often reported when the vials and balls used for ball milling are made from stainless steel. EDXRF analyses presented in Table 2 confirm a progressive contamination with these elements during the synthesis of $\mathrm{ZnFe}_{2} \mathrm{O}_{4}$. The $\mathrm{Cr}$ and Ni contamination, after $150 \mathrm{~h}$ of milling are $2.3 \%$ and $1.1 \%$, respectively. Since no other new phase was observed in the XRD patterns shown in Fig. 1, the reaction of these elements with the zinc ferrite is assumed to be due to their incorporation in solid solution in the ferrite structure during the mechano-synthesis. In addition, $\alpha$-Fe was observed from XRD pattern after $50 \mathrm{hs}$ of milling, increasing up to $1 \%$ at $150 \mathrm{~h}$ milling. This contamination is coming also from the jar.

Since disordered zinc ferrite is metastable with respect to structural changes under heat treatments, zinc ferrite synthetized by $150 \mathrm{~h}$ milling was annealed for $1 \mathrm{~h}$ at temperatures up to $600{ }^{\circ} \mathrm{C}$. XRD patterns in Fig. 3 show that the shape and positions of the diffractions peaks remain unaltered up to $300^{\circ} \mathrm{C}$. A gradual narrowing of diffraction lines and redistribution of their intensities are observed for higher annealing temperatures, which can be associated to the grain growth, lattice strain relief and the vanishing of the mechanically induced inversion. The most important microstructural parameter determined from the XRD patterns using Rietveld refinement are summarized in Table 3. Table S1 in Supporting Information shows the atomic positions, distances and angles for low and high inversion degree. 
Table 2. Chemical composition (\%mass) of milled powder determined by means of XRF. The standard deviations are in parenthesis.

\begin{tabular}{|c|c|c|c|c|}
\hline \multirow{2}{*}{ Milling Time (h) } & \multicolumn{4}{|c|}{ Elements } \\
\cline { 2 - 5 } & $\mathbf{F e}$ & $\mathbf{Z n}$ & $\mathbf{C r}$ & $\mathbf{N i}$ \\
\hline $\mathbf{5}$ & $61.7(2)$ & $38.0(2)$ & $0.14(2)$ & $0.10(1)$ \\
\hline $\mathbf{2 0}$ & $61.3(2)$ & $38.0(2)$ & $0.26(3)$ & $0.15(1)$ \\
\hline $\mathbf{1 5 0}$ & $62.2(2)$ & $34.5(2)$ & $2.3(2)$ & $1.1(1)$ \\
\hline
\end{tabular}

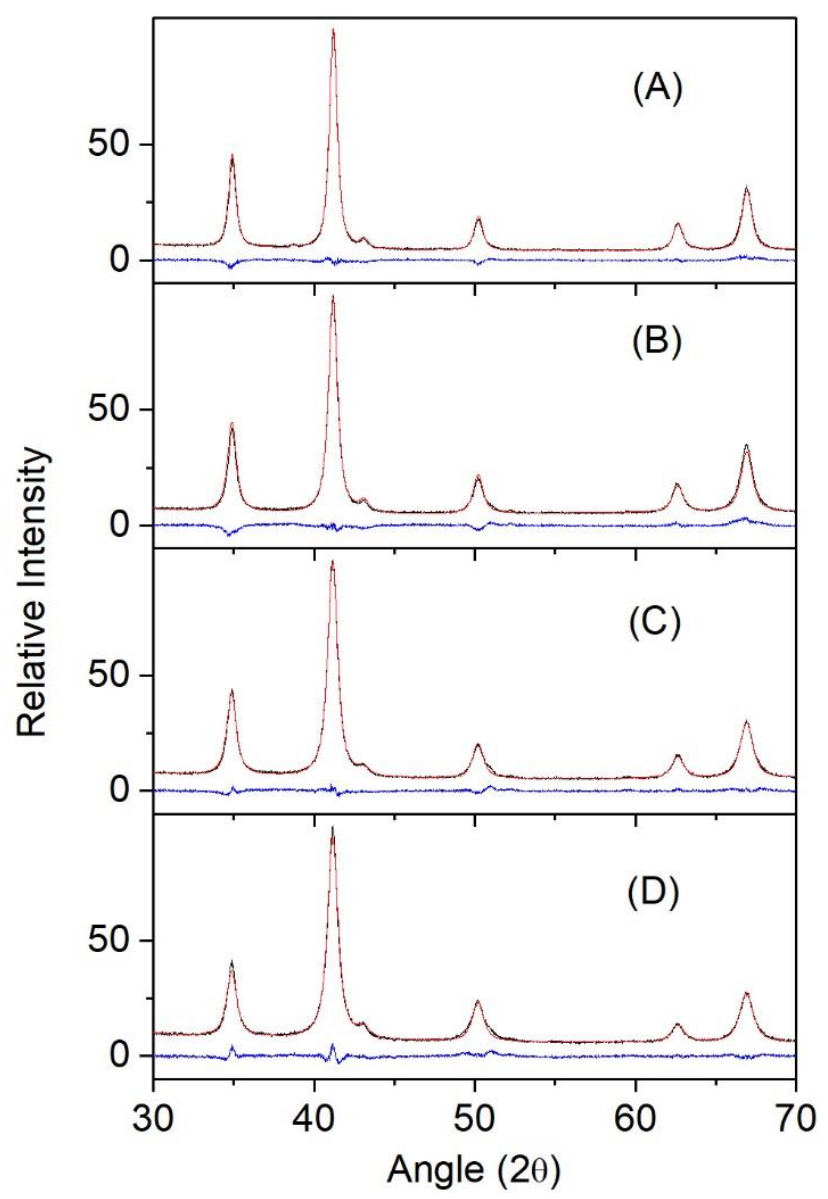

Figure 3: $\mathrm{XRD}$ patterns of $\mathrm{ZnFe}_{2} \mathrm{O}_{4}$ at different annealing temperatures and as-milled. A) $600^{\circ} \mathrm{C}$, D) $\left.500 \mathrm{C}^{\circ} \mathrm{C}\right) 400{ }^{\circ} \mathrm{C} \mathrm{D}$ ) As milled. The red line is the experimental data, black line is the fit, and blue line is the difference.

Table 3. Structural parameters and magnetic measurements as a function of annealing temperatures $\left(\mathrm{T}_{\mathrm{A}}\right)$ of $\mathrm{Zn} 150$ sample. The saturation magnetization $\mathrm{M}_{\mathrm{s}}$, coercive field $\mathrm{H}_{\mathrm{c}}$, glassing temperature $\mathrm{T}_{\mathrm{g}}$, inversion degree $\delta$, cell parameter $a$, crystallite size $d$ and $\alpha$-Fe and/or $\alpha-\mathrm{Fe}_{2} \mathrm{O}_{3}$ contamination. The standard deviations are in parenthesis.

\begin{tabular}{|c|c|c|c|c|c|c|c|}
\hline $\mathbf{T}$ & $\mathbf{M}_{\mathrm{s}}(\mathbf{e m u} / \mathrm{g})$ & $\mathbf{H c}(\mathbf{O e})$ & $\mathbf{T}_{\mathrm{g}}(\mathbf{K})$ & $\boldsymbol{\delta}$ & Lattice & Cryst. & $\alpha-\mathrm{Fe} /$ \\
\hline
\end{tabular}




\begin{tabular}{|c|c|c|c|c|c|c|c|c|c|}
\hline$\left({ }^{\circ} \mathrm{C}\right)$ & $\mathbf{5 K}$ & $\mathbf{3 0 0 K}$ & $\mathbf{5 K}$ & $\mathbf{3 0 0 K}$ & & & $\begin{array}{c}\text { paramete } \\
\mathbf{r}(\mathbf{a})\end{array}$ & $\begin{array}{c}\text { size } \\
(\mathbf{n m})\end{array}$ & $\begin{array}{c}\boldsymbol{\alpha}-\mathrm{Fe}_{2} \mathbf{O}_{\mathbf{3}} \\
(\boldsymbol{\%})\end{array}$ \\
\hline $\mathrm{RT}$ & 72.7 & 36.4 & $375(2)$ & 0 & $>300$ & $0.57(1)$ & $8.4323(5)$ & $11(1)$ & 1.3 \\
\hline 300 & 79.2 & 33.7 & $360(2)$ & 0 & 280 & $0.53(1)$ & $8.4299(5)$ & $11(1)$ & 1.3 \\
\hline 400 & 40.9 & 14.4 & $564(1)$ & 0 & 135 & $0.30(1)$ & $8.4298(5)$ & $12(1)$ & 1.0 \\
\hline 500 & 25.7 & 4.4 & $720(1)$ & 0 & 70 & $0.22(1)$ & $8.4337(5)$ & $13(1)$ & 1.0 \\
\hline 600 & 20.0 & 1.8 & $820(3)$ & 0 & 52 & $0.18(1)$ & $8.4351(5)$ & $16(2)$ & 1.2 \\
\hline
\end{tabular}

\subsection{Magnetic characterization}

\subsubsection{As milled-samples}

The evolution of the magnetic properties with the ball milling was followed by VSM measurements at room temperature over the field range of $+50 \mathrm{kOe}$ to $-50 \mathrm{kOe}$. The $\mathrm{M}-$ $\mathrm{H}$ loops of milled samples show the typical features of FM-like behavior with negligible values of coercive field $\left(\mathrm{H}_{\mathrm{C}}\right)$ and remanent magnetization $\left(\mathrm{M}_{\mathrm{R}}\right)$ at zero fields, as shown in Fig 4. The shape of the $\mathrm{M}(\mathrm{H})$ curves is about the same for all the samples with a gradual increase of the saturation magnetization $\left(\mathrm{M}_{\mathrm{s}}\right)$ with the milling time as zinc ferrite phase increases.

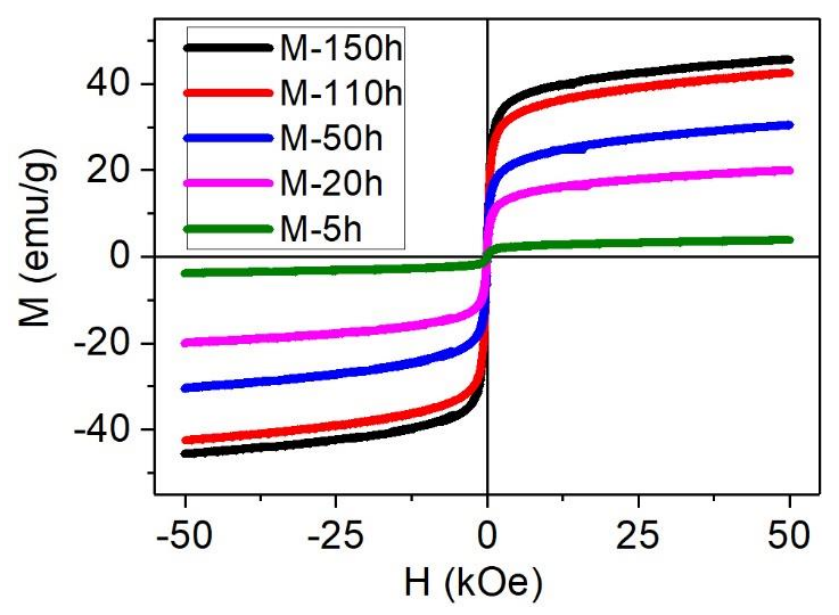

Figure 4: Hysteresis loops of milled samples from 5 to 150 hours of milling. The magnetization is per gram of sample. All measurements are a $300 \mathrm{~K}$.

Although the overall increase in magnetization with milling time may be attributed to a higher amount of spinel zinc ferrite with a random distribution of $\mathrm{Zn}^{+2}$ and $\mathrm{Fe}^{+3}$ cations, the presence of $\alpha-\mathrm{Fe}_{2} \mathrm{O}_{3}$ in the intermediate stages of transformation process introduces additional complexity in the interpretation because this phase is weakly ferromagnetic at 
room temperature. After $5 \mathrm{~h}$ milling, no evidence of zinc ferrite is observed by XRD; therefore, the magnetization is given only by hematite with $\sim 66 \%$ of the total mass (see Fig. 1B) and saturation magnetization can be calculated as $M_{s} \sim 0.4 \mathrm{emu} / \mathrm{g}$. After $10 \mathrm{~h}$ milling, the $\mathrm{ZnFe}_{2} \mathrm{O}_{4}$ becomes to synthesize and saturation magnetization can be calculated by considering the mass percent of the ferrite since contribution from hematite can be discarded due to the almost neglecting saturation magnetization. ${ }^{43}$ The saturation magnetization of zinc ferrite, $\mathrm{M}_{\mathrm{s}}\left[\mathrm{ZnFe}_{2} \mathrm{O}_{4}\right]$, can be calculated by dividing the experimental magnetic results by the zinc ferrite mass obtained from XRD. Figure 5 shows the evolution of the $\mathrm{M}_{\mathrm{s}}\left[\mathrm{ZnFe}_{2} \mathrm{O}_{4}\right]$ as a function of milling time (contribution from hematite is almost neglecting).

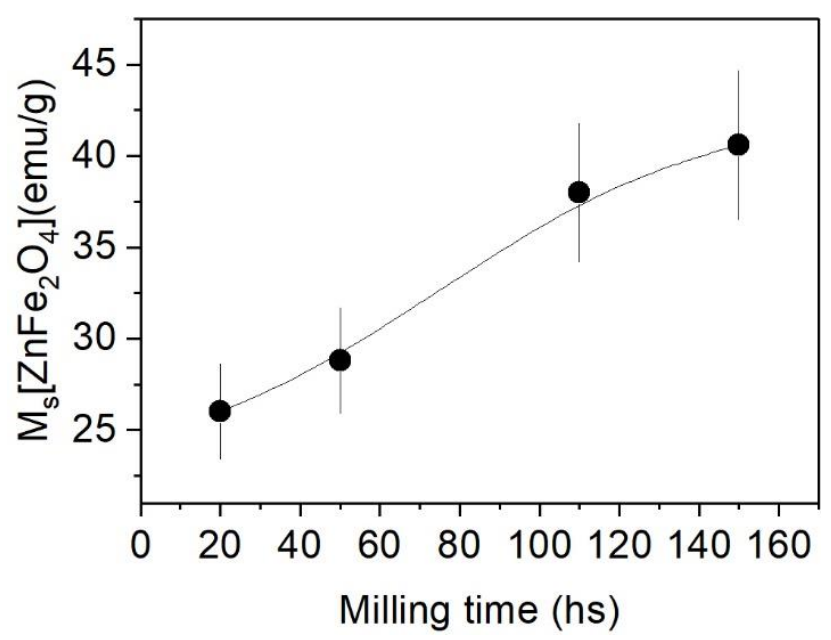

Figure 5: $\mathrm{M}_{\mathrm{s}}$ of spinel phase as function of the milling time. The magnetization is per gram of $\mathrm{ZnFe}_{2} \mathrm{O}_{4}$ obtained from the Rietveld fits (see Table 1).

\subsubsection{Annealed samples}

The magnetic properties of zinc ferrite are related to many factors, such as particle shape and size, structure, etc., which are influenced by the synthesis method and the processing route. The as milled single phase sample has a large inversion degree $\delta \sim 0.6$, therefore, successive annealing at 300, 400, 500 and $600{ }^{\circ} \mathrm{C}$ during $1 \mathrm{~h}$ allows investigating the relation between microstructure and magnetic properties of the zinc ferrite. As can be seen from Table 3, the crystallite size varies from 11 to $16 \mathrm{~nm}$ with thermal treatment. Therefore, it can be assumed that the variation of the crystallite size due to annealing play a minor role in the magnetic properties. 
As shown in Fig 6, the hysteresis loops show the coexistence of two contributions: one given by a linear dependence of the magnetization due to the presence of a paramagnetic phase, and the other one with FM-like behavior. The former is the responsible of the lack of saturation at high field, and the second one is observable at low field region (see Fig. 6D). ${ }^{28}$ FM-like behavior denotes here any ferro-, ferri- or/and superparamagnetic contribution that saturates at high fields. In order to know the saturation magnetization of the FM-like contribution, the paramagnetic one can be subtracted in the following way:

The susceptibility $\chi$ is the sum of all magnetic contributions, paramagnetic (PM), diamagnetic (DM), AFM and FM-like and can be described as follow:

$$
\chi=\chi_{P M}+\chi_{D M}+\chi_{A F M}+\chi_{F M}
$$

On the other hand, at high fields (HF), only the paramagnetic, diamagnetic and AFM orderings vary with field since the others are saturated. ${ }^{44}$ Therefore, the FM-like contributions can be discarded at HF, and high field susceptibility, $\chi \mathrm{HF}$, can be described as:

$$
\chi_{H F}=\chi_{D M}+\chi_{P M}+\chi_{A F M}
$$



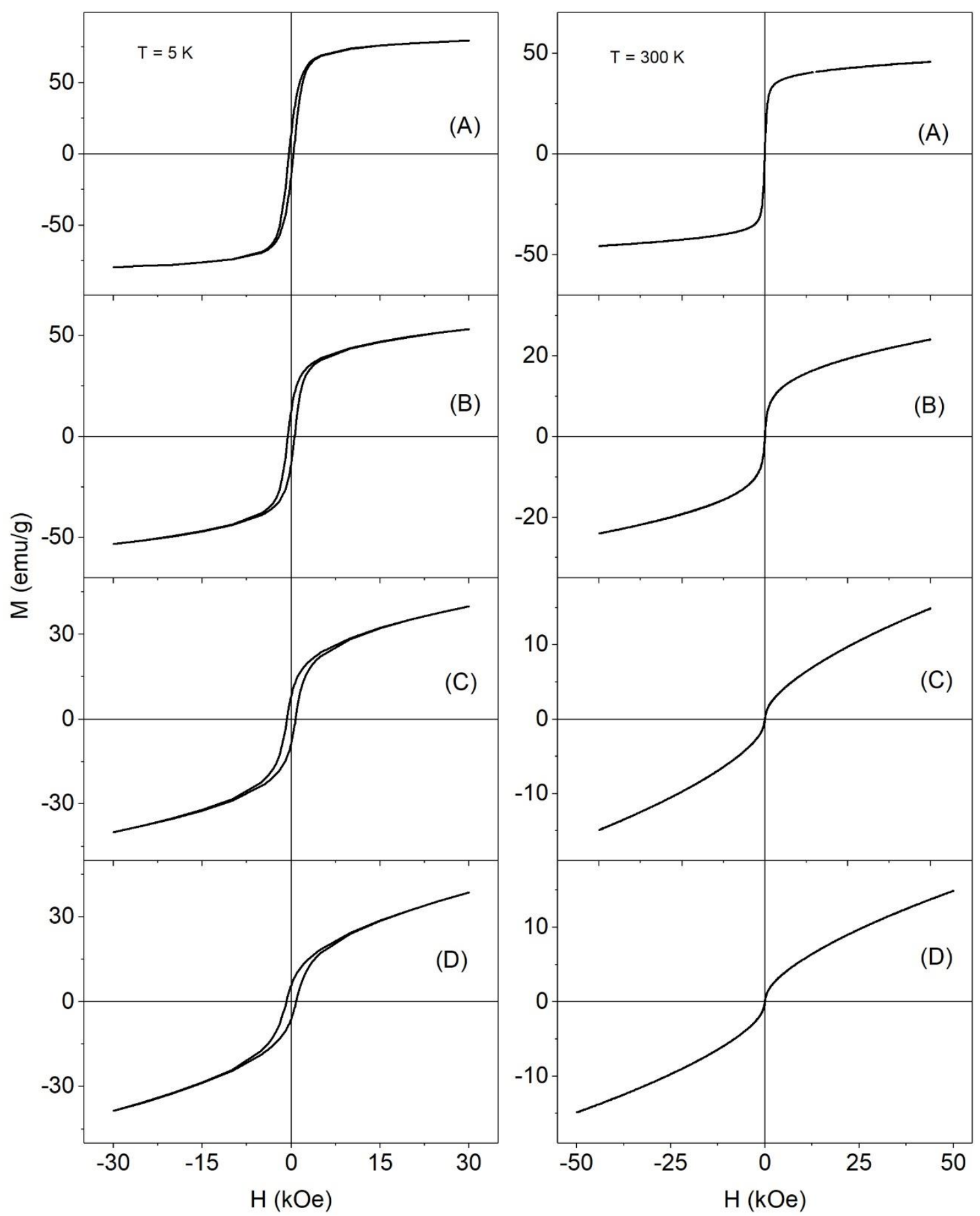

Figure 6: $\mathrm{M}(\mathrm{H})$ curves of $\mathrm{Zn} 150$ at $5 \mathrm{~K}$ (left panel) and $300 \mathrm{~K}$ (right panel) at different annealing temperatures: (A) as-milled sample, (B) $400{ }^{\circ} \mathrm{C}$; (C) $500{ }^{\circ} \mathrm{C}$ and (D) $600{ }^{\circ} \mathrm{C}$.

Consequently, the ferri-, ferro- and/or superparamagnetic contributions can be evaluated by calculating $\chi_{\mathrm{HF}}$ and subtracting it to the whole magnetization curve. In this way, the saturation magnetization $\mathrm{M}_{\mathrm{s}}$ of the FM-like contribution can be calculated for all the samples (see Table 3). 
In contrast to the PM behavior of bulk sample, the as milled sample shows a FM-like behavior even at room temperature, as shown in Fig. 6. As annealing temperature increases, the magnetization curves show a monotonously decreasing saturation magnetization plus an increasing PM contribution. At room temperature, $\mathrm{M}_{\mathrm{s}}$ decreases from $40 \mathrm{emu} / \mathrm{g}$ in as-milled powder to $1.8 \mathrm{emu} / \mathrm{g}$ after annealing at $600{ }^{\circ} \mathrm{C}$. On the other hand, at low temperature, there appears a hysteresis loops completely saturated with $\mathrm{M}_{\mathrm{s}}$ $=72 \mathrm{emu} / \mathrm{g}$ (see Fig. $6 \mathrm{~A}$ and table 3 ) for as-milled samples, whereas at $400{ }^{\circ} \mathrm{C}$ annealing a small AFM contribution shows up at high field and it increases with annealing temperature.

The smallest $\mathrm{H}_{\mathrm{C}}$ value at $5 \mathrm{~K}$ (see Table 3 and Fig. 7) corresponds to the as-milled samples with almost saturated magnetization at room temperature (can be seen from Fig. 6A (right panel)). However, as $\delta$ decreases (annealing temperature increases), the PM contribution appearing at room temperature (Fig. 6B, right panel) is accompanied by an increase of $\mathrm{Hc}$ at $5 \mathrm{~K}$ (Fig. 7). This PM contribution is a consequence of the ordering of $\mathrm{Zn}$ and $\mathrm{Fe}$ atoms in the normal spinel which gives an AFM ordering bellow Neel temperature. This paramagnetic (or AFM) contribution at room temperature (or at $5 \mathrm{~K}$ ) increases as $\delta$ decreases and it is accompanied by an increase of $\mathrm{H}_{\mathrm{C}}$.

As can be seen from Fig. 7, Hc and Mr/Ms decrease with increasing inversion degree. For $\delta<0.3$, the $\mathrm{Mr} / \mathrm{Ms}$ values indicate that the particles are random distributed and they are affected by dipolar interactions; whereas Hc decreases with the increase of FiM fraction. For high inversion degree, $\delta>0.5$, the FiM fraction is the highest and the $\mathrm{Mr} / \mathrm{Ms}$ the smallest one, suggesting that the demagnetizing factor can contribute in a considerable way for this range of inversion degree. 

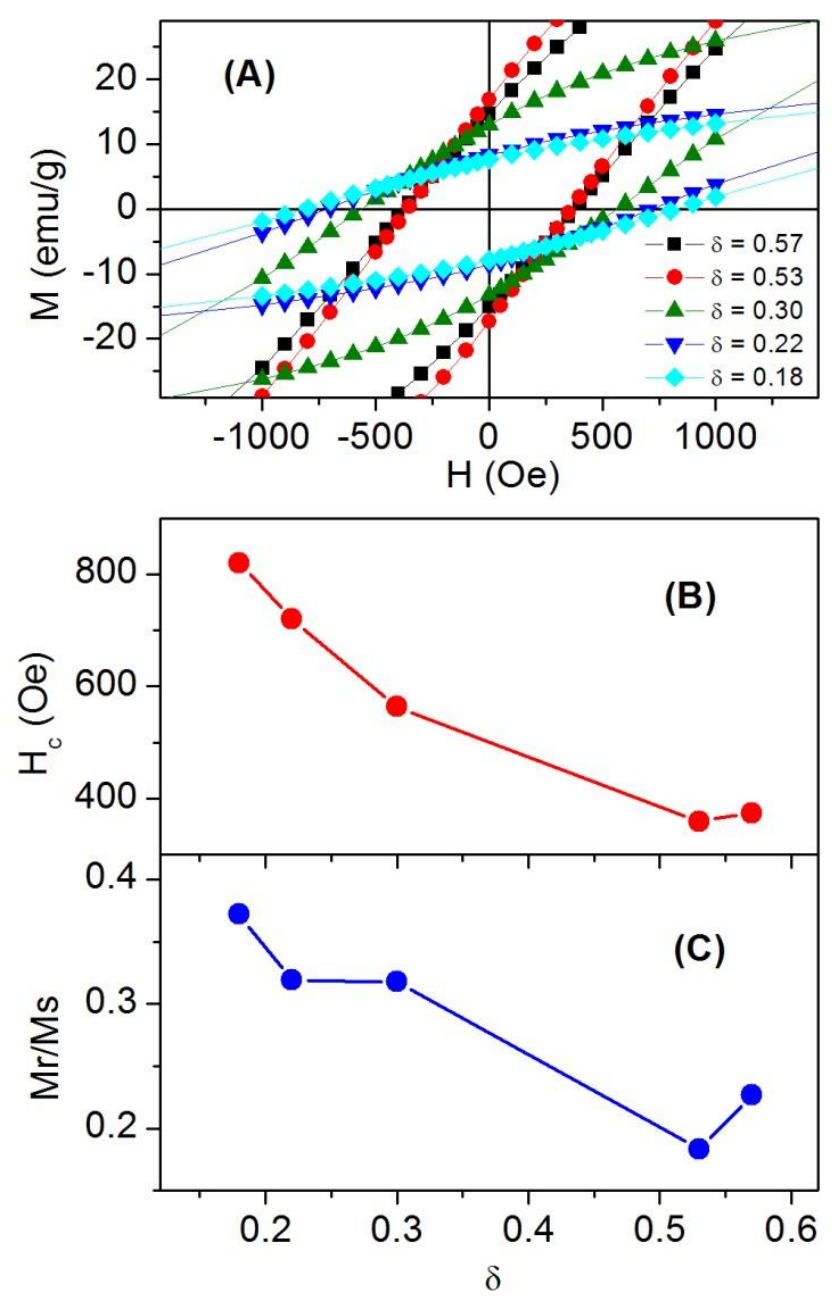

Figure 7: Details of the hysteresis loops at $5 \mathrm{~K}$ as a function of inversion degree (A). The coercive field $\mathrm{Hc}(\mathrm{B})$ and the remanence $\mathrm{Mr} / \mathrm{Ms}(\mathrm{C})$ at $5 \mathrm{~K}$ as a function of inversion degree $\delta$.

Since a rather moderate increase of the crystallite size was determined from XRD line broadening analysis (from $\sim 11 \mathrm{~nm}$ in as milled material to $\sim 16 \mathrm{~nm}$ after annealing at $600^{\circ} \mathrm{C}$ ) and the critical size corresponding to the transition from multidomain to singledomain regions for $\mathrm{ZnFe}_{2} \mathrm{O}_{4}$ has been reported to be ranging from 15 to $18 \mathrm{~nm}$, ${ }^{45}$ the $\mathrm{H}_{\mathrm{C}}$ increase cannot be assigned to the particle size increase but rather to the inversion parameter decrease, which is the most important parameter determining the transition from FM-like to $\mathrm{PM}$ behavior in nano-sized $\mathrm{ZnFe}_{2} \mathrm{O}_{4}$ particles.

To further clarify the nature of magnetic behavior, the temperature dependence of the magnetization under ZFC-FC procedure was measured. As shown in Fig. 8, there is a clear difference between the thermal dependence of ZFC-FC curves with the annealing temperature. The ZFC curves present maximum at different temperatures that depend on 
annealing temperature. Since the particles are of nanometric size, this maximum could correspond to a blocking temperature $\left(\mathrm{T}_{\mathrm{B}}\right)$ in the case of SPM behavior or a glass temperature $\left(\mathrm{T}_{\mathrm{g}}\right)$ in the case of spin glass behavior. As can be seen, this maximum decreases as annealing temperature increases, i.e., as grain size slightly increases. This behavior is opposite to a blocked superparamagnetic state whose $\mathrm{T}_{\mathrm{B}}$ increases with grain size, consequently, this maximum corresponds to a glassy magnetic state, which has been reported by other authors for long time milled samples. ${ }^{46} \mathrm{~T}_{\mathrm{g}}$ is determined by the thermal energy required to overcome the superexchange interactions and represents the average temperature at which the magnetic moments of the nanoparticles are blocked during the measurement time. Figure 8 shows broad distribution of $\mathrm{T}_{\mathrm{g}}$ with a clear dependence on the annealing temperature, decreasing progressively from $300 \mathrm{~K}$ in the as-milled powder to $52 \mathrm{~K}$ after annealing at $600{ }^{\circ} \mathrm{C}$. The decrease of $\mathrm{T}_{\mathrm{g}}$ is correlated to the decrease of inversion degree: as the inversion degree decreases, more $\mathrm{Zn}^{2+}$ occupies tetrahedral $\mathrm{A}$ sites and $\mathrm{Fe}^{3+}$ interacts antiferromagnetically, modifying the spin glass behavior.

From an experimental point of view, spin glasses show very peculiar and interesting nonequilibrium behaviors. One of these is the observation of memory effects: a spin glass is able to "remember" certain features of its past history in a rather remarkable way. Consequently, the memory effect is an experimental signature of spin glass systems. $^{47}$ 


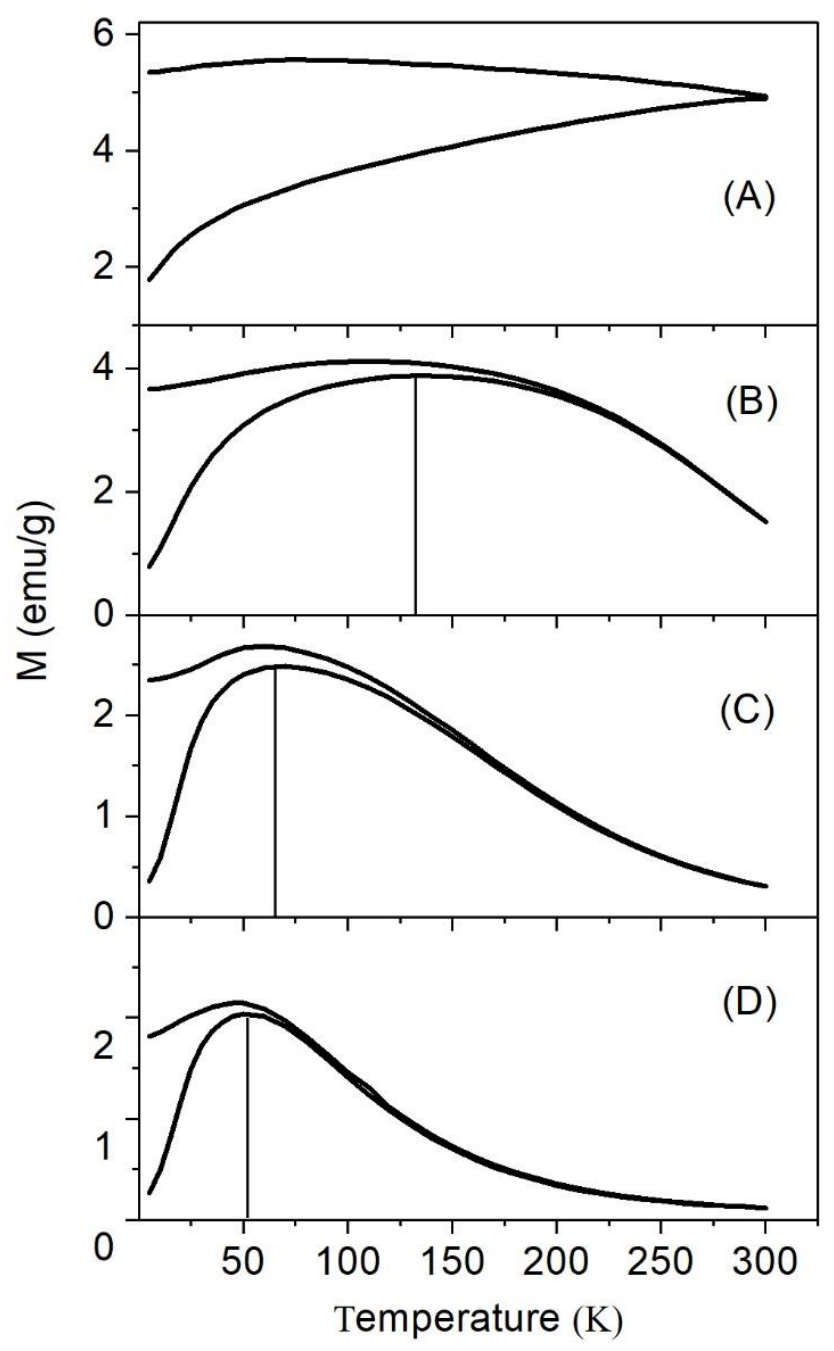

Figure 8: ZFC-FC curves of Zn150: (A) as-milled samples and annealed at (B) $400^{\circ} \mathrm{C}$, (C) 500 ${ }^{\circ} \mathrm{C}$, and (D) $600{ }^{\circ} \mathrm{C}$. Vertical lines indicate the $\mathrm{T}_{\mathrm{g}}$ : (A) $\mathrm{T}_{\mathrm{g}}>300 \mathrm{~K}$, (B) $\mathrm{T}_{\mathrm{g}}=134 \mathrm{~K}$, (C) $\mathrm{T}_{\mathrm{g}}=70$ $\mathrm{K}$ and (D) $\mathrm{T}_{\mathrm{g}}=52 \mathrm{~K}$.

Aging and memory experiments were performed under FC protocols. In the FC memory protocol, the samples annealed at 500 and $600^{\circ} \mathrm{C}$ were cooled down under $\mathrm{H}=50$ Oe with intermittent stops at 100, 75, 50, 35 and $20 \mathrm{~K}$. At each stop, the field was switched off for $10^{4} \mathrm{~s}$, then returned to 50 Oe before cooling resumed. After this step-cooling procedure, the field was on and the magnetization was measured while increasing the temperature continuously at a fixed speed. In the case of sample annealed at $600{ }^{\circ} \mathrm{C}$, the resulting magnetization curve shows memory effects characterized by an inflection at those temperatures at which the sample has been aged, as shown in Fig. 9. This memory of each step that occurred during the cooling demonstrate that a spin glass order has been established on the material during each stage of aging. ${ }^{48}$ In the case of sample 
annealed at $500{ }^{\circ} \mathrm{C}$, no steps were observed, suggesting that the spin glass of this sample is almost neglecting.

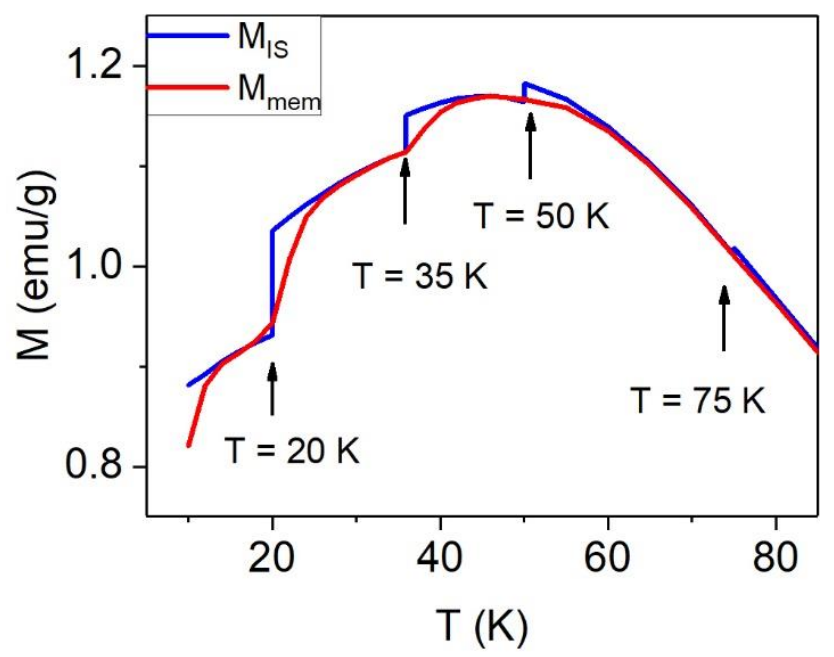

Figure 9: FC memory effect characterization by an inflection in the heating curve $\left(\mathrm{M}_{\mathrm{mem}}\right)$ at the temperatures at which the sample has been aged $\left(\mathrm{M}_{\mathrm{IS}}-\right.$ intermittent stop cooling magnetization). Sample annealed at $600{ }^{\circ} \mathrm{C}$.

\section{Discussion}

In ferrites, the magnetic interactions are strongly dependent on the type and distribution of metal cations in the crystal lattice. The cation distribution in the tetrahedral and octahedral sites in partially inverse spinel ferrites can be deduced on the basis of both Rietveld refinement of XRD data and the intensity ratio in the Mössbauer spectra of the sextets due to $\mathrm{Fe}^{3+}$ cations in the tetrahedral and octahedral sites. Taking into account that combined XRD and Mössbauer spectral studies partially inverse spinel ferrites have concluded that the cation distribution obtained from the X-ray intensity data matches very well with the cation distribution estimated from Mössbauer data, ${ }^{49-52}$ and that the diffraction profile analysis with the Rietveld method allows simultaneously getting the relevant information of the material microstructure, it was selected the use of $\mathrm{X}$ ray diffraction for characterizing the microstructural evolution during annealing.

A determining factor of the magnetic properties of mechanical-activated zinc ferrite is the lattice parameter. The FiM behavior of disordered zinc ferrite starts to be visible after $10 \mathrm{~h}$ of milling, with a high inversion degree as soon as the ferrite phase forms. Further milling leads to a progressive increase of the mass fraction of zinc ferrite 
manifested by the gradual increase of both, the intensity of diffraction peak of this phase and the relative weight of the FiM component in the $\mathrm{M}-\mathrm{H}$ loops. Rietveld refinements of the XRD patterns show that while inversion degree and crystallite size of the mechanosynthesized zinc ferrite are almost independent on the milling time, the lattice parameter and $\mathrm{M}_{\mathrm{s}}$ for the spinel phase change continuously up to $150 \mathrm{~h}$ milling (see Fig. S1).

As shown in Fig. 5, $\mathrm{M}_{\mathrm{s}}$ per gram of $\mathrm{ZnFe}_{2} \mathrm{O}_{4}$ at room temperature increases from 26 up to $40 \mathrm{emu} / \mathrm{g}$. This increase cannot be assigned to sample contamination during milling since $\mathrm{Ni}$ and $\mathrm{Cr}$ are the majority contaminations, with almost $1.1 \%$ of $\mathrm{Ni}$ and $2.3 \% \mathrm{Cr}$ after $150 \mathrm{~h}$ milling (see table 2). Considering a contribution from metallic $\mathrm{Ni}$ (less probable due to size) with $\mathrm{M}_{\mathrm{s}}=55 \mathrm{emu} / \mathrm{g}$ at room temperature, ${ }^{53}$ the magnetic contribution to the hysteresis loops is around $0.5 \mathrm{emu} / \mathrm{g}$, and cannot justify the $33 \%$ increase of $\mathrm{M}_{\mathrm{s}}$ from 20 to $150 \mathrm{~h}$ milling time. Besides, the amount of impurities cannot explain the variation of the lattice parameter. On the other hand, the contamination with $1 \%$ of $\alpha-\mathrm{Fe}$ cannot account for this difference since considering the saturation magnetization at room temperature of this impurity, $\mathrm{M}_{\mathrm{s}}=217 \mathrm{emu} / \mathrm{g}$, it therefore contributes with only $2.2 \mathrm{emu} / \mathrm{g}$ to the whole magnetization, it means that only $3 \%$ of the total magnetization of $\mathrm{ZnFe}_{2} \mathrm{O}_{4}$ could be originated by the $\alpha$-Fe jar contamination.

The evolution of these lattice parameter and $\mathrm{M}_{\mathrm{s}}$ with milling time can be assigned to the relative abundance of hematite and $\mathrm{ZnO}$ with increasing milling time. As shown in Fig. $1 \mathrm{~B}$, the content of $\mathrm{ZnO}$ was reduced more rapidly than that of hematite with increasing milling time, and after $50 \mathrm{~h}$ milling all the $\mathrm{ZnO}$ reflections disappeared completely while the amount of unreacted hematite is about $15.5 \%$. Some authors have explained this phenomenon considering that, beside the reaction $\mathrm{ZnO}+\mathrm{Fe}_{2} \mathrm{O}_{3} \rightarrow \mathrm{ZnFe}_{2} \mathrm{O}_{4}$, amorphization of some amount of $\mathrm{ZnO}$ takes place during grinding. ${ }^{29}, 54$ However, a fixed composition of the spinel at $\mathrm{Zn} / \mathrm{Fe}=0.5$ could not explain that up to $50 \mathrm{~h}$ milling the lattice parameter of this phase increases continuously from 8.4409 to $8.4435 \AA$ (see Fig. S1 from SI). Considering previously reported results for this material prepared through different synthesis methods, it can be concluded that a non-equilibrium processing may result in the occupancy of the octahedral sites by large amount of $\mathrm{Zn}^{+2}$ favoring the formation of Zn-rich ferrite spinel. ${ }^{21-22, ~ 25, ~ 27, ~ 29, ~} 55$ Makovec et al. have reported that for a single phase spinel structure the lattice parameter increases linearly 
with increasing the $\mathrm{Zn} / \mathrm{Fe}$ ratio up to a value of $\mathrm{Zn} / \mathrm{Fe}=0.8 .^{21}$ Thus, the changes in the lattice parameter of the zinc ferrite suggest that the composition of the spinel changes during the milling process getting a ratio different to 1:1 from the Rietveld refinements of the mass fraction of $\mathrm{ZnO}$ and $\alpha-\mathrm{Fe}_{2} \mathrm{O}_{3}$. A $\mathrm{Zn}$-rich spinel also explains the low $\mathrm{M}_{\mathrm{s}}$ per mass of zinc ferrite for times shorter than $50 \mathrm{~h}$ (Fig. 5) since the magnetization also reflects the $\mathrm{Zn}^{+2}$ and $\mathrm{Fe}^{+3}$ ratio cations between tetrahedral and octahedral sublattice. When the $\mathrm{Zn} / \mathrm{Fe}$ ratio is higher than 0.5 , the $\mathrm{Zn}^{+2}$ in excess incorporates in both, tetrahedral and octahedral sites. Under this condition, the resulting magnetic moment is smaller than that corresponding to the stoichiometric phase. After $150 \mathrm{~h}$ milling, all reflections of starting precursors completely disappear and then zinc ferrite reaches its stoichiometric composition $(\mathrm{Zn} / \mathrm{Fe}=0.5)$

XRD measurements revealed that increasing milling time results in the progressive formation of a phase with a spinel structure characterized by broad diffraction lines. The crystallite size in the ball-milled $\mathrm{ZnFe}_{2} \mathrm{O}_{4}$ powders was estimated to be almost independent on the milling time, reaching a value of about $\sim 11 \mathrm{~nm}$ in good agreement with that observed from the HRTEM images (see Table 1 and Fig. 2). Zinc ferrite prepared by the conventional ceramic method adopts the normal spinel structure, but cationic disordering can be induced mechanically. It has been previously reported that zinc ferrite can be synthetized by ball-milling with a partial or almost pure inverse spinel structure or samples in which both normal and inverse spinel structures can coexist. However, our Rietveld refinements of the XRD patterns of the high-energy ball-milled $\mathrm{Fe}_{2} \mathrm{O}_{3} / \mathrm{ZnO}$ mixtures show that the zinc ferrite presents always an almost complete randomization of both $\mathrm{Zn}^{+2}$ and $\mathrm{Fe}^{+3}$ cations into tetrahedral and octahedral sites of the spinel. This random cationic distribution determines the presence of $\mathrm{Fe}^{3+}$ ions in both tetrahedral and octahedral sublattices, resulting in a magnetic interaction between cations at both sites ( $\mathrm{AB}$ interactions).

The interaction between cations in A and B sites is a superexchange interaction that depends on distances and angles between magnetic cations. ${ }^{1}$ The basic characteristic of spinel, and that justifies the FiM ordering of spins, is that the exchange integrals $\mathrm{J}_{\mathrm{AA}}$, $\mathrm{J}_{\mathrm{AB}}$ and $\mathrm{J}_{\mathrm{BB}}$ are negative, that is, the exchanges are AFM. For ferrites with magnetic cations at $\mathrm{A}$ and $\mathrm{B}$ sites, the interaction $\mathrm{AB}$ is the strongest, and the magnetic spins at $\mathrm{A}$ sites order antiparallel to those in B sites; consequently, the A sites order parallel to 
each other and the same for the B sites, giving place to FiM order. In the case of normal $\mathrm{ZnFe}_{2} \mathrm{O}_{4}$ (inverse parameter $\delta=0$ ), the non-magnetic $\mathrm{Zn}$ occupies the site $\mathrm{A}$ and, then, $\mathrm{Fe}^{3+}$ cations in $\mathrm{B}$ sites order antiferromagnetically, as it is known. If $\delta \mathrm{Fe}^{3 *}$ cations migrate to A sites $(0<\delta<2 / 3)$, there appears an exchange interaction between $\mathrm{A}$ and $\mathrm{B}$ sites which is stronger than $\mathrm{AA}$ and $\mathrm{BB}$ interactions. Does it necessarily affect the interaction of the rest of non-magnetic A sites surrounded by $\mathrm{Fe}^{3+}$ cations?

Figure $10 \mathrm{~A}$ shows $\mathrm{M}_{\mathrm{s}}$ at $5 \mathrm{~K}$ as a function of inversion degree $\delta$ calculated from the hysteresis curves at high fields, after subtraction of AFM or PM contributions. As can be seen, $\mathrm{M}_{\mathrm{s}}$ increases with inversion degree, as expected, but it goes down for $\delta>0.5$.

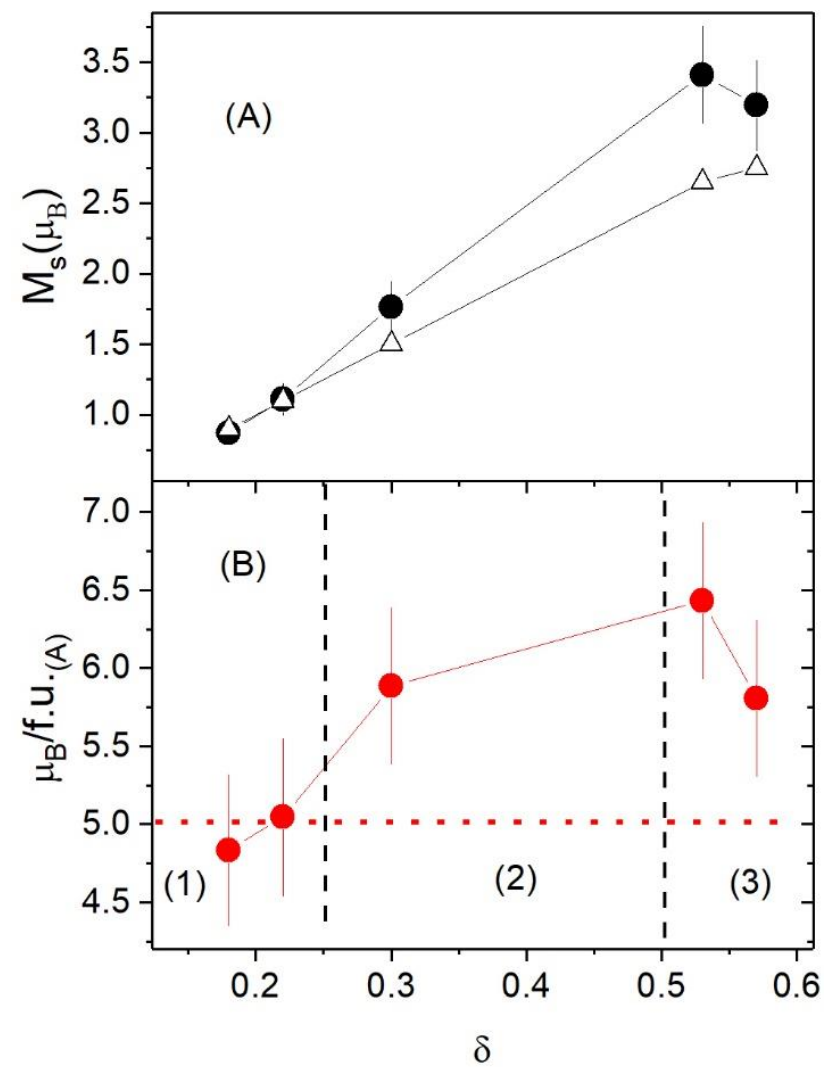

Figure 10: (A) Saturation magnetization per Bohr magneton as a function of inversion degree $\delta$ (black circles) and saturation calculated as $M_{s}=\delta \cdot 5 \mu_{B}$ (open triangles). (B) Saturation magnetization per ferromagnetic unit (f.u.) at A sites. The regions (1), (2) and (3) indicate FiM+AFM+SG, FiM and FiM+AFM phases, respectively. 
The main issue is to know the magnetic arrangement in the cell when $\mathrm{Fe}^{3+}$ sits at $\mathrm{A}$ sites. Let us consider that a $\mathrm{Fe}^{3+}$ at $\mathrm{A}$ site contribute with $5 \mu_{\mathrm{B}}$; therefore, $\mathrm{M}_{\mathrm{s}}$ has to be proportional to $\delta$ which counts for the ratio of $\mathrm{Fe}^{3+}$ in A sites. As can be seen in Fig. $10 \mathrm{~A}, \mathrm{M}_{\mathrm{s}}$ varies linearly with $\delta$ for low inversion degree but the slope increases as soon as $\delta>0.25$. By calculating how many Bohr magnetons per $\mathrm{Fe}^{3+}$ atoms (ferromagnetic units, f.u.) placed in A site there are, it is observed $5 \mu_{\mathrm{B}} /$ f.u. for low $\delta$ but it increases for higher inversion degree (Fig. 10B). A priori, it is not possible to have higher contribution than that coming from $\mathrm{Fe}^{3+}$ at A sites.

To try to understand where this extra contribution is coming from, it is necessary to understand the atomic arrangement and their contributions. In the spinel structure, the cations in $\mathrm{A}$ sites are surrounding by four $\mathrm{O}^{2-}$ which, in turn, are bonding to $\mathrm{B}$ cations (see scheme 1). To figure out this spatial arrangement, a 2D network square with a similar configuration of the spinel ferrite is shown in Scheme 2. This network only shows the spatial distribution of cations without the oxygen atoms and represents half of the crystallographic structure. Scheme $2 \mathrm{~A}$ illustrates the normal spinel with $\mathrm{Zn}^{2+}$ at the center of the tetrahedral A sites (blue circles) and $\mathrm{Fe}^{3+}$ at the center of octahedral $\mathrm{B}$ sites (black arrows). The dotted arrows represent the $\mathrm{J}_{\mathrm{AA}}, \mathrm{J}_{\mathrm{BB}}$ and $\mathrm{J}_{\mathrm{AB}}$ exchange interactions.

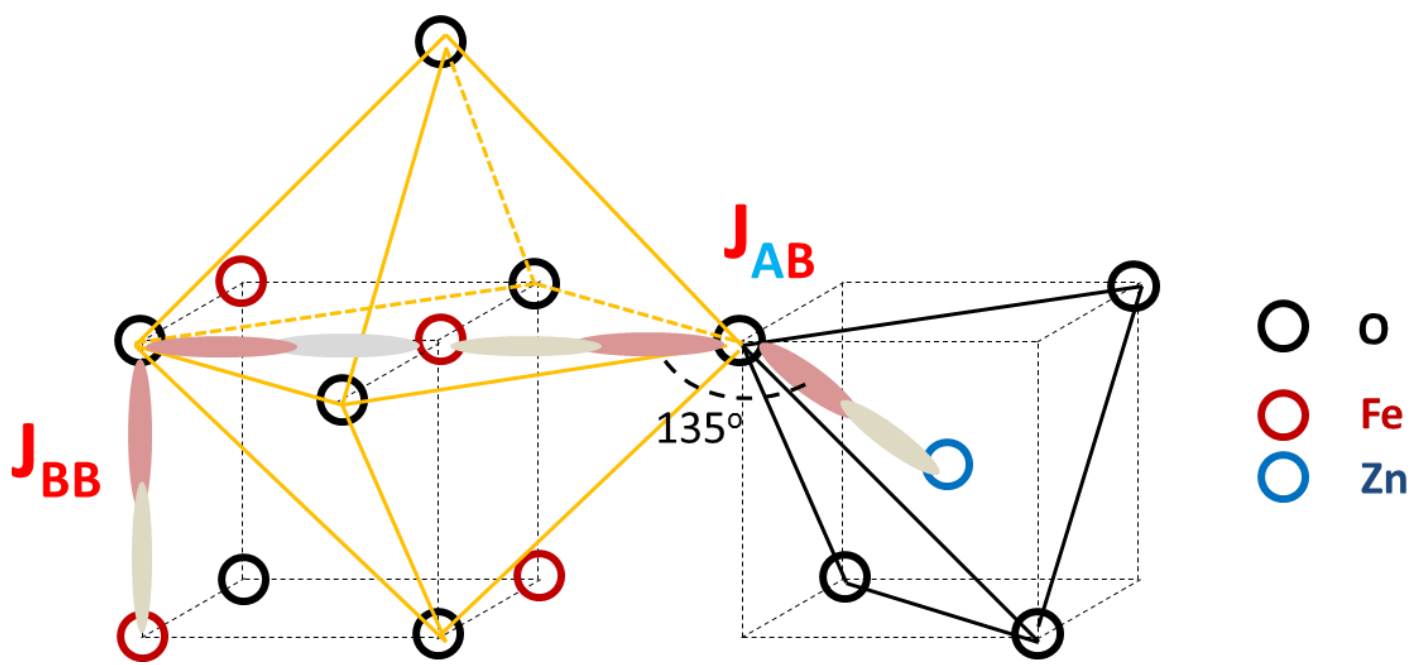

Scheme 1: Representation of the tetrahedral and octahedral sites in spinel structure with the ideal subtended angle. In this scheme, the blue circle represents the non-magnetic $\mathrm{Zn}^{2+}$ atom and the red ones the magnetic $\mathrm{Fe}^{3+}$ atom, respectively. The $\mathrm{O}^{2-}$ anions are the corner. The $\mathrm{J}_{\mathrm{BB}}$ and $\mathrm{J}_{\mathrm{AB}}$ the exchange integrals of sites $\mathrm{BB}$ and $\mathrm{AB}$, respectively. 
Let us assume that one of the four $\mathrm{Fe}^{3+}$ in B sites migrates to the A site (Scheme 2(B)). The remaining three $\mathrm{Fe}^{3+}$ of the $\mathrm{B}$ sites (the fourth neighbor is a non-magnetic $\mathrm{Zn}$ ) form a triangular ordering with AFM interactions giving place to geometrical magnetic frustration since the simple 'up-down' spin alignments cannot be satisfied with all antiparallel magnetic bonds. ${ }^{47}$ The other remaining A sites are occupied by non-magnetic $\mathrm{Zn}$ and their surrounding B sites could order AFM. Consequently, this configuration gives $5 \mu_{B}$ per $\mathrm{A}$ site occupied by $\mathrm{Fe}^{3+}$ cations and $0 \mu_{B}$ for the rest. At low inversion degree, the saturation magnetization is proportional to the numbers of $\mathrm{Fe}^{3+}$ exchanged from B to A sites: $M_{s}=\delta \cdot 5 \mu_{B}$

As site exchange increases, there appear squares with non-magnetic atoms in A sites which surrounding is polarized by the next A neighbors with magnetic cations and make spin glass behavior goes missing, thus improving the net magnetic moment of the unit cell (see Scheme 2C). In this case it appears two non-compensated AFM sublattice that gives place to FiM interactions. As a result, the net magnetization is higher than $5 \mu_{B}$ per $\mathrm{Fe}^{3+}$ at $\mathrm{A}$ sites because these sites are occupied by non-magnetic $\mathrm{Zn}$.

Finally, if $\delta>0.5$, i.e., there are sites with two magnetic and two non-magnetic corners and, then, it appears compensated AFM interactions between A and B sites resulting in $m_{A B}=0 \mu_{B}$. Consequently, the total magnetization decreases because the coexistence of AFM and FiM ordering.

The maximum inversion degree cannot exceed $2 / 3$ since entropy reaches a maximum at this value; $\delta>2 / 3$ would imply a new order and entropy should decrease, which is forbidden. An hypothetical case of $\delta=1,(\mathrm{Fe})[\mathrm{ZnFe}] \mathrm{O}_{4}$, would produce a system with AFM interactions but now given by superexchange interactions between $\mathrm{Fe}^{3+}$ at $\mathrm{A}$ sites with $\mathrm{Fe}^{3+}$ in $\mathrm{B}$ sites, i.e., $\mathrm{J}_{\mathrm{AB}}$ exchange. 
(A)

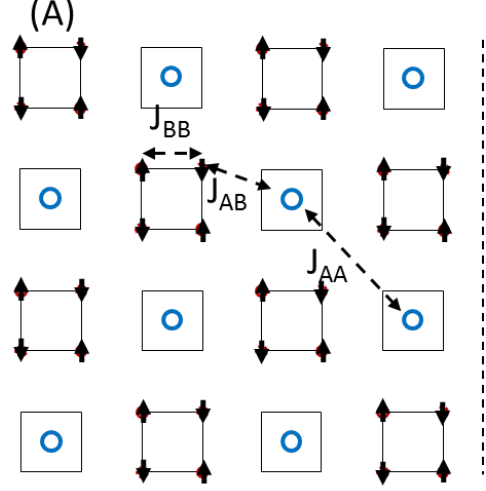

(B)

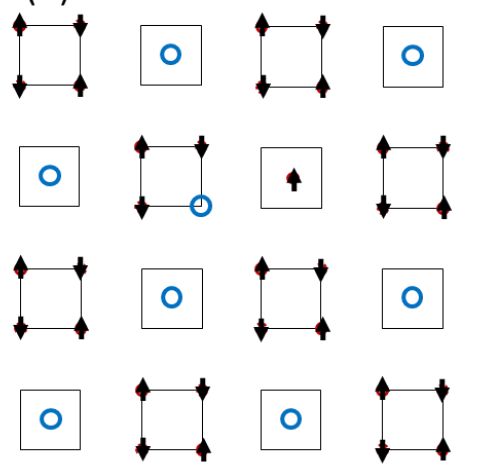

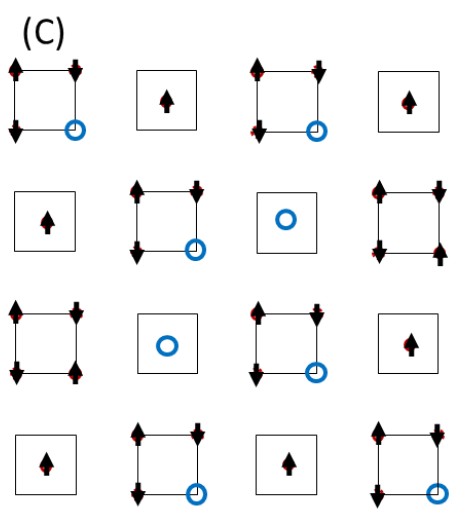

Scheme 2: Scheme of a square network of non-magnetic atoms (blue circles) and magnetic atoms (arrows) that represents half of the crystallographic cell. For the sake of clarity, the anionic network has been eliminated and only the cations are represented. (A) Normal ferrite with the $\mathrm{Zn}$ and $\mathrm{Fe}^{3+}$ atoms at $\mathrm{A}$ and $\mathrm{B}$ sites, respectively, with $\mathrm{AFM}$ interactions. (B) $\mathrm{Zn}$ and $\mathrm{Fe}^{3+}$ have exchanged their positions, this promote geometrical magnetic frustration that gives place to spin glass behavior. (C) As the number of exchanged atoms increases, the spin glass contribution decreases and non-compensate AFM interactions appear giving place to FiM interactions.

This is accordance with our experimental results, where the magnetic behavior can be divided in three regions depending on the inversion degree: 1) for $0<\delta<1 / 4$, hysteresis loops are the sum of FiM and AFM interactions indicating these two magnetic phases coexist inside the cell, additionally, the ratio FiM to AFM contributions decreases with $\delta$. This is also reflected by the changes in $\mathrm{H}_{\mathrm{C}}$ with the inversion degree. On the other hand, spin glass behavior is observed in this region. 2) for $1 / 4<\delta<1 / 2$, the magnetization curves are mainly FiM with neglecting AFM or spin glass contributions. The magnetization is higher than the $5 \mu \mathrm{B}$ that provides $\mathrm{Fe}^{3+}$ in A sites. This suggests that there exist A sites with non-magnetic atoms which are also contributing to the whole magnetization. This is possible if the surrounding A sites with $\mathrm{Fe}^{3+}$ polarize the $\mathrm{A}$ sites with non-magnetic $\mathrm{Zn}^{2+}$. In this region, there isn't any spin glass behavior because geometrical spin frustration vanishes. 3) for $1 / 2<\delta<2 / 3$ the whole magnetization decreases and the $\mathrm{H}_{\mathrm{C}}$ increases slightly, which could suggest that the coexistence of FiM and AFM interactions. This onset of AFM is given by $\mathrm{Fe}^{3+}$ in $\mathrm{A}$ sites with only two $\mathrm{Fe}^{3+}$ at the corners leading to a compensated AFM interaction and producing a decreases of the whole magnetization and an increase of the $\mathrm{H}_{\mathrm{C}}$ due to the interaction between both magnetic phases.

It can be concluded that the inversion degree provides three regions where there exist different magnetic phases (see Fig. 10B): 1) low inversion degree with coexistence of FiM, AFM and spin glass, 2) medium inversion degree with FiM provided by A sites 
with non-magnetic atoms polarized by their neighbors, 3) high inversion degree with FiM and AFM coexistence, where this AFM is given by compensated spins in the $\mathrm{J}_{\mathrm{AB}}$ interactions.

\section{Conclusions}

It has been possible to relate the magnetic properties on the structural and microstructural parameters of the $\mathrm{Zn}$ ferrite synthesized by ball-milling. Thus, the magnetic behavior depends mainly on the cationic inversion of the $\mathrm{Fe}^{3+}$ when occupying tetrahedral positions of the spinel. The cationic inversion degree can be modified by a non-equilibrium process path, obtaining a different magnetic behavior in a nanometer crystallite size.

From XRD and Rietveld analysis it is observed that $\mathrm{Zn}$ ferrite with maximum cationic disorder close to 0.6 (random) is obtained by mechano-synthesis. During this process, the evolution of the precursor phases and the ferrite is not homogeneous, forming first a $\mathrm{Zn}$ rich ferrite (with $\mathrm{Zn}: \mathrm{Fe}>0.5$ ) to finally reach the stoichiometry after several hours of milling, as determined by magnetic characterization and Rietveld analysis. It is worth noting that the zinc ferrite has a high inversion degree as soon as the phase is formed.

This highly disordered ferrite can be partially ordered by annealing one hour at different temperatures, so that its inversion parameter can be varied from very high to very low values. The as-milled samples have an inversion degree of 0.57 and it decreases to 0.18 for annealing at $600 \mathrm{C}$ during $1 \mathrm{~h}$. At this point, ZFC-FC curves show the maximum spin glass behavior of the samples with $\mathrm{T}_{\mathrm{g}}=50 \mathrm{~K}$.

The main result of this work is the determination of different magnetic behaviors as a function of the inversion degree. A first regime, associated to low inversion degree ( $0<\delta<1 / 4$ ), shows FiM, AFM and spin glass coexistence where the site exchange of $\mathrm{Zn}^{2+}$ and $\mathrm{Fe}^{3+}$ promotes FiM interactions but also spin glass due to geometrical spin frustration, in addition to AFM ordering. A second regime, with medium inversion degree $(1 / 4<\delta<1 / 2)$, where FiM interactions are dominant and spin glass behavior has vanished. This regime has the highest magnetization since, apart from the A sites with magnetic cations, there exist A sites with no magnetic cations where FiM is induced by the surrounding neighbors. A third regimen, with $1 / 2<\delta<2 / 3$, where 
AFM contributions are present once again since, due to the high inversion degree, there appears $\mathrm{Fe}^{3+}$ in $\mathrm{A}$ sites surrounded by only two shared $\mathrm{Fe}^{3+}$ in octahedral sites. This configuration gives place to compensated AFM contributions of the type $\mathrm{J}_{\mathrm{AB}}$ with a decrease of the wole magnetization. To sum up, the zinc ferrite has a mainly FiM behavior at $\delta=0.5$ with the highest magnetization $m=6.5 \mu_{B}$ per $\mathrm{Fe}^{3+}$ in A site.

Therefore, it can be concluded that the maximum magnetization for $\mathrm{Zn}$ ferrite could be close to $\delta=0.5$, and it decreases for higher and smaller inversion degree. A more detailed investigation around $\delta=0.5$ is needed in order to confirm these results.

\section{Corresponding Author}

* Patricia de la Presa, pmpresa@ucm.es, to whom correspondence should be addressed.

\section{Author Contributions}

The manuscript was written through contributions of all authors. All authors have given approval to the final version of the manuscript.

Supporting Information: additional structural parameters information.

\section{ACKNOWLEDGMENT}

This work was supported by grants from the Spanish Ministry of Science and Innovation MAT2015- 67557-C2-1-P.

\section{ABBREVIATIONS}

FM: ferromagnetism; AFM: antiferromagnetism; FiM: ferrimagnetism; PM: paramagnetism

\section{REFERENCES}


1. Goldman, A., Modern Ferrite Technology, 2nd Edition; Springer +Business media: New York, 2006.

2. Sugimoto, M., The Past, Present, and Future of Ferrites. Journal of the American Ceramic Society 1999, 82, 269-280.

3. Kotnala, R. K.; Shah, J., Chapter 4 - Ferrite Materials: Nano To spintronics Regime. In Handbook of Magnetic Materials, Buschow, K. H. J., Ed. Elsevier: 2015; Vol. 23, pp 291-379.

4. Hu, G.; Choi, J. H.; Eom, C. B.; Harris, V. G.; Suzuki, Y., Structural Tuning of the Magnetic Behavior in Spinel-Structure Ferrite Thin Films. Physical Review B 2000, 62, R779-R782.

5. Lavina, B.; Salviulo, G.; Giusta, A. D., Cation Distribution and Structure Modelling of Spinel Solid Solutions. Physics and Chemistry of Minerals 2002, 29, 10-18.

6. Šepelák, V.; Bergmann, I.; Feldhoff, A.; Heitjans, P.; Krumeich, F.; Menzel, D.; Litterst, F. J.; Campbell, S. J.; Becker, K. D., Nanocrystalline Nickel Ferrite, Nife2o4: Mechanosynthesis, Nonequilibrium Cation Distribution, Canted Spin Arrangement, and Magnetic Behavior. The Journal of Physical Chemistry C 2007, 111, 5026-5033.

7. Stewart, S. J.; Figueroa, S. J. A.; Lopez, J. M.; Marchetti, S. G.; Bengoa, J. F.; Prado, R. J.; Requejo, F. G., Cationic Exchange in Nanosized Znfe204 Spinel Revealed by Experimental and Simulated near-Edge Absorption Structure. Physical Review B 2007, 75, 4.

8. Upadhyay, C.; Verma, H. C.; Sathe, V.; Pimpale, A. V., Effect of Size and Synthesis Route on the Magnetic Properties of Chemically Prepared Nanosize Znfe204. Journal of Magnetism and Magnetic Materials 2007, 312, 271-279.

9. Oneill, H. S., Temperature Dependence of the Cation Distribution in Zinc Ferrite (Znfe2o4) from Powder Xrd Structural Refinements. Eur. J. Mineral. 1992, 4, 571-580.

10. Szotek, Z.; Temmerman, W. M.; Ködderitzsch, D.; Svane, A.; Petit, L.; Winter, H., Electronic Structures of Normal and Inverse Spinel Ferrites from First Principles. Physical Review B 2006, 74, 174431.

11. Oneill, H. S. C.; Navrotsky, A., Simple Spinels - Crystallographic Parameters, Cation Radii, Lattice Energies and Cation Distribution. American Mineralogist 1983, 68, 181-194.

12. Kalendova, A.; Vesely, D., The Properties of Znfe204 as an Anticorrosion Pigment Dependent Upon the Structure of Initial Fe2o3. Anti-Corrosion Methods and Materials 2008, $55,175-190$.

13. Zhang, H. J.; Meng, F. N.; Liu, L. Z.; Chen, Y. J.; Wang, P. J., Highly Sensitive H2s Sensor Based on Solvothermally Prepared Spinel Znfe2o4 Nanoparticles. J. Alloy. Compd. 2018, 764, 147-154.

14. Xu, Y. G.; Liu, Q. Q.; Xie, M.; Huang, S. Q.; He, M. Q.; Huang, L. Y.; Xu, H.; Li, H. M., Synthesis of Zinc Ferrite/Silver lodide Composite with Enhanced Photocatalytic Antibacterial and Pollutant Degradation Ability. J. Colloid Interface Sci. 2018, 528, 70-81.

15. Rani, M.; Shanker, U., Photocatalytic Degradation of Toxic Phenols from Water Using Bimetallic Metal Oxide Nanostructures. Colloids and Surfaces a-Physicochemical and Engineering Aspects 2018, 553, 546-561.

16. Sahu, T. K.; Shah, A. K.; Gogoi, G.; Patra, A. S.; Ansari, M. S.; Qureshi, M., Effect of Surface Overlayer in Enhancing the Photoelectrochemical Water Oxidation of in Situ Grown One-Dimensional Spinel Zinc Ferrite Nanorods Directly onto the Substrate. Chemical Communications 2018, 54, 10483-10486.

17. Yadav, N. G.; Chaudhary, L. S.; Sakhare, P. A.; Dongale, T. D.; Patil, P. S.; Sheikh, A. D., Impact of Collected Sunlight on Znfe204 Nanoparticles for Photocatalytic Application. J. Colloid Interface Sci. 2018, 527, 289-297.

18. Rodriguez, K. L. S.; Stewart, S. J.; Zelis, P. M. M.; Pasquevich, G. A.; Torres, C. E. R., Role of Defects on the Magnetic Behaviour of the Geometrically Frustrated Spinel Znfe2o4. J. Alloy. Compd. 2018, 752, 289-295.

19. Stewart, S. J.; Al-Omari, I. A.; Sives, F. R.; Widatallah, H. M., Non-Equilibrium Cation Influence on the Neel Temperature in Znfe2o4. J. Alloy. Compd. 2010, 495, 506-508. 
20. Yao, C. W.; Zeng, Q. S.; Goya, G. F.; Torres, T.; Liu, J. F.; Wu, H. P.; Ge, M. Y.; Zeng, Y. W.; Wang, Y. W.; Jiang, J. Z., Znfe2o4 Nanocrystals: Synthesis and Magnetic Properties. Journal of Physical Chemistry C 2007, 111, 12274-12278.

21. Makovec, D.; Kodre, A.; Arcon, I.; Drofenik, M., The Structure of Compositionally Constrained Zinc-Ferrite Spinel Nanoparticles. Journal of Nanoparticle Research 2011, 13, 1781-1790.

22. Mozaffari, M.; Arani, M. E.; Amighian, J., The Effect of Cation Distribution on Magnetization of Znfe2o4 Nanoparticles. Journal of Magnetism and Magnetic Materials 2010, $322,3240-3244$.

23. Hu, X.; Guan, P.; Yan, X., Hydrothermal Synthesis of Nano-Meter Microporous Zinc Ferrite. China Particuology 2004, 2, 135-137.

24. Swamy, P. M. P.; Basavaraja, S.; Lagashetty, A.; Rao, N. V. S.; Nijagunappa, R.; Venkataraman, A., Synthesis and Characterization of Zinc Ferrite Nanoparticles Obtained by Self-Propagating Low-Temperature Combustion Method. Bulletin of Materials Science 2011, 34, 1325-1330.

25. Ammar, S.; Jouini, N.; Fievet, F.; Stephan, O.; Marhic, C.; Richard, M.; Villain, F.; Moulin, C. C. D.; Brice, S.; Sainctavit, P., Influence of the Synthesis Parameters on the Cationic Distribution of Znfe204 Nanoparticles Obtained by Forced Hydrolysis in Polyol Medium. Journal of Non-Crystalline Solids 2004, 345, 658-662.

26. Bid, S.; Pradhan, S. K., Preparation of Zinc Ferrite by High-Energy Ball-Milling and Microstructure Characterization by Rietveld's Analysis. Mater. Chem. Phys. 2003, 82, 27-37.

27. Ehrhardt, H.; Campbell, S. J.; Hofmann, M., Structural Evolution of Ball-Milled Znfe2o4. J. Alloy. Compd. 2002, 339, 255-260.

28. Lopez-Maldonado, K. L.; de la Presa, P.; Betancourt, I.; Mancilla, J. R. F.; Aquino, J. A. M.; Hernando, A.; Galindo, J. T. E., Superparamagnetic Response of Zinc Ferrite Incrusted Nanoparticles. J. Alloy. Compd. 2015, 637, 443-448.

29. Nachbaur, V.; Tauvel, G.; Verdier, T.; Jean, M.; Juraszek, J.; Houvet, D., Mecanosynthesis of Partially Inverted Zinc Ferrite. J. Alloy. Compd. 2009, 473, 303-307. 30. Sato, T.; Haneda, K.; Seki, M.; lijima, T., Morphology and Magnetic Properties of Ultrafine Znfe204 Particles. Applied Physics a-Materials Science \& Processing 1990, 50, 13-16. 31. Oliver, S. A.; Hamdeh, H. H.; Ho, J. C., Localized Spin Canting in Partially Inverted Znfe2o4 Fine Powders. Physical Review B 1999, 60, 3400-3405.

32. Li, F. S.; Wang, L.; Wang, J. B.; Zhou, Q. G.; Zhou, X. Z.; Kunkel, H. P.; Williams, G., Site Preference of $\mathrm{Fe}$ in Nanoparticles of Znfe2o4. Journal of Magnetism and Magnetic Materials 2004, 268, 332-339.

33. Nakashima, S.; Fujita, K.; Tanaka, K.; Hirao, K.; Yamamoto, T.; Tanaka, I., First-Principles Xanes Simulations of Spinel Zinc Ferrite with a Disordered Cation Distribution. Physical Review B 2007, 75.

34. Suryanarayana, C., Mechanical Alloying and Milling. Progress in Materials Science 2001, 46, 1-184.

35. Koch, K. C., Mechanical Milling and Alloying. In Materials Science and Technology, Cahn, R. W.; Haasen, P.; Kramer, E. J., Eds. 2006.

36. Villars, P.; Cenzual, K., Pearson's Crystal Data: Crystal Structure Database for Inorganic Compounds. ASM International ${ }^{\circledR}$ : Materials Park, Ohio, USA, 2017/2018.

37. Oneill, H. S., Temperature-Dependence of the Cation Distribution in Zinc Ferrite (Znfe2o4) from Powder Xrd Structural Refinaments. Eur. J. Mineral. 1992, 4, 571-580.

38. Balzar, D., et al., Size-Strain Line-Broadening Analysis of the Ceria Round-Robin Sample. Journal of Applied Crystallography 2004, 37, 911-924.

39. Andersson, M. S.; De Toro, J. A.; Lee, S. S.; Normile, P. S.; Nordblad, P.; Mathieu, R., Effects of the Individual Particle Relaxation Time on Superspin Glass Dynamics. Physical Review B 2016, 93. 
40. Jonason, K.; Vincent, E.; Hammann, J.; Bouchaud, J. P.; Nordblad, P., Memory and Chaos Effects in Spin Glasses. Physical Review Letters 1998, 81, 3243-3246.

41. Khurshid, H.; Lampen-Kelley, P.; Iglesias, O.; Alonso, J.; Phan, M. H.; Sun, C. J.; Saboungi, M. L.; Srikanth, H., Spin-Glass-Like Freezing of Inner and Outer Surface Layers in Hollow Gamma-Fe2o3 Nanoparticles. Scientific Reports 2015, 5.

42. Sahoo, S.; Petracic, O.; Kleemann, W.; Nordblad, P.; Cardoso, S.; Freitas, P. P., Aging and Memory in a Superspin Glass. Physical Review B 2003, 67.

43. Ahmadzadeh, M.; Romero, C.; McCloy, J., Magnetic Analysis of Commercial Hematite, Magnetite, and Their Mixtures. AIP Advances 2018, 8, 056807.

44. Richter, C.; van der Pluijm, B. A., Separation of Paramagnetic and Ferrimagnetic Susceptibilities Using Low Temperature Magnetic Susceptibilities and Comparison with High Field Methods. Physics of the Earth and Planetary Interiors 1994, 82, 113-123.

45. Blanco-Gutiérrez, V.; Torralvo-Fernández, M. J.; Sáez-Puche, R., Magnetic Behavior of Znfe204 Nanoparticles: Effects of a Solid Matrix and the Particle Size. The Journal of Physical Chemistry C 2010, 114, 1789-1795.

46. Procopio, E. F.; Larica, C.; Muniz, E. P.; Litterst, F. J.; Passamani, E. C., Cluster-Glass-Like Behavior in Zinc Ferrite Nanograins. Journal of Magnetism and Magnetic Materials 2018, 467, 1-7.

47. Mydosh, J. A., Spin Glasses: Redux: An Updated Experimental/Materials Survey. Rep. Prog. Phys. 2015, 78, 25.

48. Dupuis, V.; Bert, F.; Bouchaud, J. P.; Hammann, J.; Ladieu, F.; Parker, D.; Vincent, E., Aging, Rejuvenation and Memory Phenomena in Spin Glasses. Pramana-J. Phys. 2005, 64, 1109-1119.

49. Gupta, M. P.; Mathur, H. B., The Cation Distribution in the Ferrite Fev204:Mossbauer and X-Ray Diffraction Studies. Journal of Physics C: Solid State Physics 1975, 8, 370-376.

50. Singhal, S.; Barthwal, S. K.; Chandra, K., Structural, Magnetic and Mössbauer Spectral Studies of Nanosize Aluminum Substituted Nickel Zinc Ferrites. Journal of Magnetism and Magnetic Materials 2006, 296, 94-103.

51. Singhal, S.; Barthwal, S. K.; Chandra, K., Xrd, Magnetic and Mössbauer Spectral Studies of Nano Size Aluminum Substituted Cobalt Ferrites (Coalxfe2-Xo4). Journal of Magnetism and Magnetic Materials 2006, 306, 233-240.

52. Kumar, G.; Kotnala, R. K.; Shah, J.; Kumar, V.; Kumar, A.; Dhiman, P.; Singh, M., Cation Distribution: A Key to Ascertain the Magnetic Interactions in a Cobalt Substituted Mg-Mn Nanoferrite Matrix. Physical Chemistry Chemical Physics 2017, 19, 16669-16680.

53. Crangle, J.; Goodman, G. M., The Magnetization of Pure Iron and Nickel. Proceedings of the Royal Society of London. Series A, Mathematical and Physical Sciences 1971, 321, 477-491.

54. Kim, W.; Saito, F., Mechanochemical Synthesis of Zinc Ferrite from Zinc Oxide and AFe203. Powder Technology 2001, 114, 12-16.

55. Prithviraj Swamy, P. M.; Basavaraja, S.; Lagashetty, A.; Srinivas Rao, N. V.; Nijagunappa, R.; Venkataraman, A., Synthesis and Characterization of Zinc Ferrite Nanoparticles Obtained by Self-Propagating Low-Temperature Combustion Method. Bulletin of Materials Science 2011, 34, 1325-1330. 


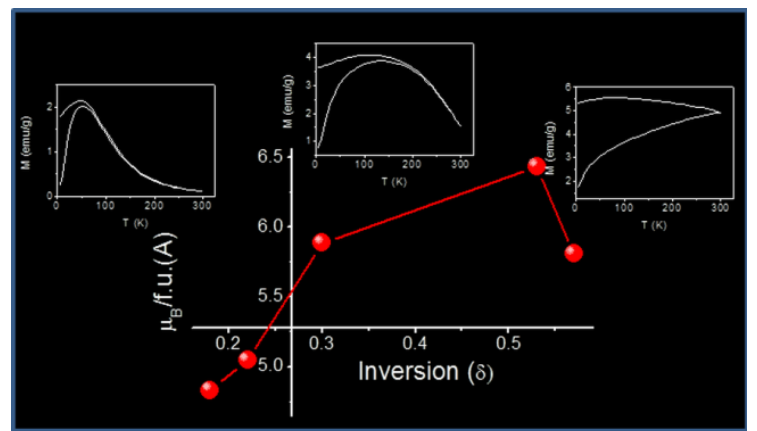

TOC Graphic 